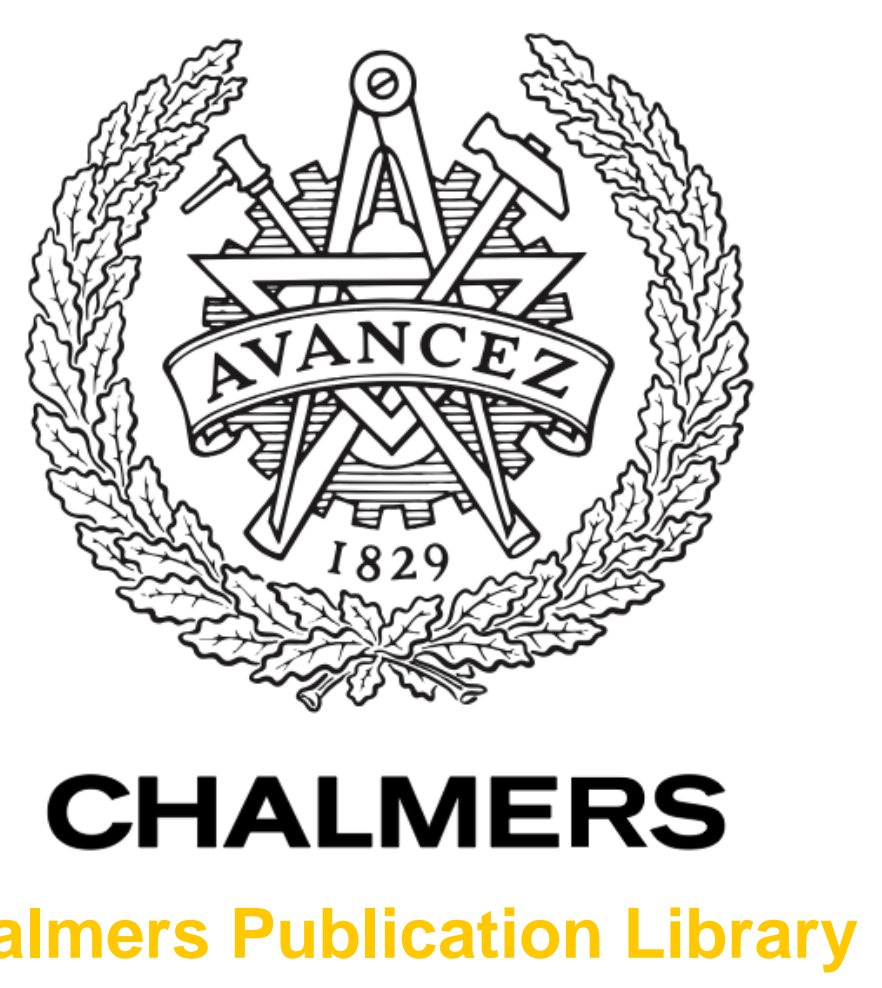

Chalmers Publication Library

\title{
Comparative Analysis of Unipolar and Bipolar Control of Modular Battery for Thermal and State-of-Charge Balancing
}

This document has been downloaded from Chalmers Publication Library (CPL). It is the author's version of a work that was accepted for publication in:

IEEE Transactions on Vehicular Technology (ISSN: 0018-9545)

Citation for the published paper:

Altaf, F. ; Egardt, B. (2016) "Comparative Analysis of Unipolar and Bipolar Control of Modular Battery for Thermal and State-of-Charge Balancing". IEEE Transactions on

Vehicular Technology

http://dx.doi.org/10.1109/TVT.2016.2587720

Downloaded from: http://publications.lib.chalmers.se/publication/231165

Notice: Changes introduced as a result of publishing processes such as copy-editing and formatting may not be reflected in this document. For a definitive version of this work, please refer to the published source. Please note that access to the published version might require a subscription. 


\title{
Comparative Analysis of Unipolar and Bipolar Control of Modular Battery for Thermal and State-of-Charge Balancing
}

\author{
Faisal Altaf and Bo Egardt, Fellow, IEEE,
}

\begin{abstract}
Thermal and state-of-charge imbalance is a well known issue to cause nonuniform ageing in batteries. The modular battery based on cascaded converters is a potential solution to this problem. This paper presents bipolar control (BPC) of a modular battery and compares it with previously proposed unipolar control (UPC) mode in terms of thermal/SOC balancing performance and energy efficiency. The BPC needs four-quadrant operation of full-bridge converter using bipolar pulse-width modulation (PWM) inside each module, whereas UPC only needs half-bridge converter with unipolar PWM. The BPC, unlike UPC, enables charging of some cells while discharging others. An averaged state-space electro-thermal battery model is derived for a convex formulation of the balancing control problem. The control problem is formulated on a constrained LQ form and solved in a model predictive control framework using one-step ahead prediction. The simulation results show that BPC, without even requiring load current variations, gives better balancing performance than UPC, but at the cost of reduced efficiency. The UPC requires at least current direction reversal for acceptable balancing performance. In short, the UPC is a more cost and energy efficient solution for $\mathrm{EV}$ and PHEV applications whereas the BPC can be beneficial in applications involving load cycles with high current pulses of long duration.
\end{abstract}

Index Terms-Modular battery, SOC balancing, thermal balancing, multilevel converters, averaging, model predictive control.

\section{INTRODUCTION}

The electrification and hybridization of vehicle powertrain is being vastly adopted by automotive industry to increase fuel efficiency and to meet ever decreasing exhaust emission limits. The Lithium-ion battery is one of the major alternative power sources currently being considered for this purpose. The battery pack of these electrified/hybridized vehicles (xEVs) is one of the most expensive, but a key component in the powertrain. Therefore, the battery lifetime is an important factor for the success of xEVs. The conventional battery system in $\mathrm{xEVs}$ consists of long string of series connected modules along with $\mathrm{dc} / \mathrm{dc}$ converter for dc-link voltage regulation as shown in Fig. 1. Due to the fixed series connection, the same current passes through all the modules. This is a socalled uniform duty operation (UDO) of cells. If modules have nonuniform state-of-health (parametric variations) then they may suffer from unequal stress and energy drain under

Copyright (c) 2015 IEEE. Personal use of this material is permitted. However, permission to use this material for any other purposes must be obtained from the IEEE by sending a request to pubs-permissions @ieee.org

Manuscript received December 14, 2015; revised March 19, 2016: accepted June 13, 2016. Date of publication XX XX 2016; date of current version July XX, 2016. Manuscript received in final form July 01, 2016. This work was supported by the Chalmers Energy Initiative, Sweden. Recommended by Associate Editor Alireza Khaligh.

F. Altaf and B. Egardt are with Chalmers University of Technology, Automatic Control Group, Department of Signals and Systems, 41296 Gothenburg Sweden (e-mails: \{faisal.altaf, bo.egardt\} @chalmers.se).
UDO, which can cripple the whole battery pack. The health and ageing rate of each Li-ion cell in a battery pack is greatly affected by various factors like state-of-charge (SOC) level, depth-of-discharge (DOD), temperature, and c-rate etc [1][4]. In short, the cells in the string being stored/cycled at higher SOC/DOD and temperature age faster than those at lower SOC/DOD and temperature. Therefore, thermal, SOC, and DOD imbalances in a battery pack may cause nonuniform ageing of cells. Another serious issue is that the cell imbalance and nonuniform ageing are tightly coupled, which may lead to a vicious cycle resulting in the premature end of battery life. In addition to nonuniform ageing, the SOC imbalance also has a detrimental impact on the total usable capacity of the battery [5], [6]. It is also worth mentioning that thermal, SOC, and DOD imbalance is inevitable in battery packs of $\mathrm{xEVs}$ due to variations in cell parameters and operating conditions, see [7] and [8]. Thus, thermal and SOC balancing is quite critical for optimal performance of automotive batteries.

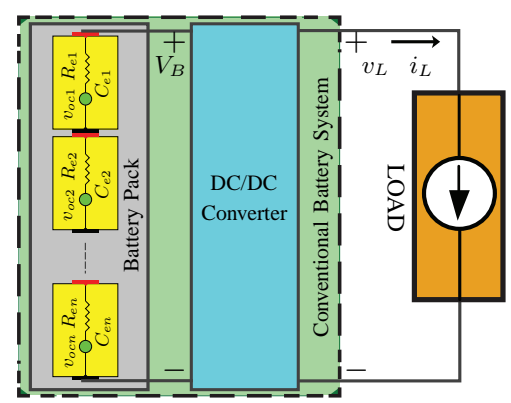

Fig. 1. Conventional battery with $n$ series-connected battery modules and dc/dc converter to regulate dc-link voltage $v_{L}$

The SOC balancing can be achieved using various types of passive or active SOC balancers, see [9]-[11], whereas thermal balancing can potentially be achieved using reciprocating airflow as proposed in [8], but not under parametric variations as shown in [12]. The notion of simultaneous thermal and SOC balancing using a single active balancing device was introduced in [12]-[14]. A similar kind of conceptual study has also been carried out in [15]. Thermal and SOC balancing are two tightly coupled and somewhat conflicting objectives, but it is possible to achieve both simultaneously in an average sense [6]. For this, load variations and surplus voltage in the battery pack are required. Also, a special balancing device that enables the non-uniform load scheduling of cells, is needed.

The modular battery system based on cascaded converters is a potential candidate for simultaneous thermal and SOC balancing purpose [12]-[14], [16], [17]. The modular battery consists of $n$ cascaded power units (PUs), each containing a smaller battery module and a dc/dc converter, which enables 
bidirectional power flow from each module. There are various $\mathrm{dc} / \mathrm{dc}$ converter topologies like full-bridge and half-bridge that can be employed inside PUs. The modular battery is reconfigurable to generate a range of terminal voltages. It provides a large redundancy in the voltage synthesis, which gives extra degrees-of-freedom in control. The concept of modular battery is also studied recently by other authors for xEVs [18]-[21] as well as for smart grid energy storage applications [22], but only SOC balancing and voltage control problems are addressed at most. The modular battery proposed in our earlier studies [16], [17] targets multiple control objectives including thermal balancing, SOC balancing, and dclink voltage regulation. This requires a more advanced control algorithm to decide power flow from each module.

The electro-thermal control problem of the modular battery can be solved using a one-step model predictive control (MPC) scheme, which requires information only about current battery power demand [16], [17]. The problem is formulated on a standard linear quadratic (LQ) form based on the decomposition of controller into two orthogonal components, one for voltage control and the other for balancing control. The voltage controller strictly satisfies the load voltage demand, distributing the demanded power almost equally among all modules. Therefore, the balancing controller corrects the power distribution by optimally exploiting the available redundancy in the modular battery to achieve thermal and SOC balancing without disturbing the voltage. However, the studies [16] and [17] were restricted to unipolar control (UPC) of modular battery. The UPC mode only needs a half-bridge converter with single unipolar pulse-width modulation (PWM) in each module, but it does not allow polarity inversion of battery cells. Therefore, there is no possibility to charge some cells while discharging others. Due to this, the simultaneous balancing of temperature and SOC may become a daunting task for one-step MPC under aggressive drive cycles like US06 and constant high speed driving [16]. This is mainly due to their aggressive nature (high $c$-rate) and lower level of variations in load current magnitude and direction compared to stop-n-go urban type driving.

In this paper, the bipolar control (BPC) of a modular battery for simultaneous thermal and SOC balancing is presented. The BPC mode needs four-quadrant operation of full-bridge converter using three-level bipolar PWM (generated using two unipolar PWMs). This allows polarity inversion (so-called negative actuation) of cells in the string, which enables charging of some cells while discharging others. Therefore, some extra freedom is achieved to control SOC and temperature of each module. The main purpose of this study is to thoroughly investigate the pros and cons of both UPC and BPC modes in terms of their balancing performance as well as energy efficiency (first contribution). For this purpose, a unified model predictive control method is devised in which UPC becomes a special case of BPC mode (second contribution). The method is tailored using a similar controller structure as proposed in [16], but it is based on a new average modeling approach, which is proposed in this study to get convex optimization problem under both UPC and BPC modes (third contribution). This is an important contribution because the averaging approach used in [12]-[14], [16], and [17] would lead to non- convex problem under BPC mode, which is hard to solve.

The comparative analysis is done in a simulation study for US06 and constant $80 \mathrm{mph}$ motorway driving cycles. The study is focussed on an air-cooled modular battery consisting of only four series-connected modules for illustration purpose. In order to analyze the effectiveness of the control modes, the cells are assumed to have significant differences in their resistances, capacities, and initial SOCs. The load on the modular battery is assumed to be three-phase electric drive of Toyota Prius PHEV running in pure EV mode.

The paper is organized as follows. Section II summarizes the notation used in this paper. Section III gives an overview of two modular battery configurations along with UPC and BPC modes. The new averaging approach and electro-thermal model of battery are presented in sections IV and V. The control problem formulation is presented in section VI. The simulation setup is presented in section VII and the performance comparison between UPC and BPC is given in section VIII. Finally, section IX concludes the paper.

\section{NotATION}

Throughout this paper, $\mathbb{R}\left(\mathbb{R}_{+}\right), \mathbb{R}^{n}\left(\mathbb{R}_{+}^{n}\right)$, and $\mathbb{R}^{n \times m}$ are used to denote set of (non-negative) real numbers, set of real vectors with $n$ (non-negative) elements, and set of real matrices with order $n \times m$ respectively. Unless otherwise noted, calligraphic letters are used to denote subsets of real vector spaces. The identity matrix of order $n \times n$, column $n$-vector of ones, column $n$-vector of zeros are denoted by $I_{n}, 1_{n}$, and $0_{n}$ respectively. The Euclidean norm and absolute value of variables are denoted by $\|\cdot\|$ and $|\cdot|$ respectively whereas $\|x\|_{Q}^{2}$ is used to denote $x^{\mathrm{T}} Q x$. The mean and standard deviation of a sequence of variable $x$ are denoted by $m_{x}$ and $\sigma_{x}$ respectively. For sake of saving space, MATLAB's notation 'diag' and 'blkdiag' is occasionally used to denote diagonal and block-diagonal matrices respectively.

\section{MOdUlar BATTERY}

\section{A. Introduction}

The modular battery, shown in Fig. 2, consists of $n$ seriesconnected power units (PUs), each containing a dc/dc power converter with ideal switches and an isolated Cell ${ }_{i}$. It supplies voltage $v_{L}(t)=\sum_{i=1}^{n} v_{L i}(t) \in\left[0, v_{L, \max }\right] \subseteq \mathbb{R}_{+}$to a variable load with current demand $i_{L}(t) \in\left[i_{L, \min }, i_{L, \max }\right] \subseteq \mathbb{R}$, where $v_{L i}$ is the terminal voltage of $\mathrm{PU}_{i}$. This modular structure enables independent control of power flow from each unit, making it suitable for cell balancing purpose.

The power flow from each $\mathrm{PU}_{i}$ is controlled using two control variables $u_{i}^{+} \in[0,1]$ and $u_{i}^{-} \in[0,1]$ (so-called positive and negative duty cycles, see section IV for details). These control variables are fed into a pulse width modulator, which generates unipolar switching functions $s_{i}^{+} \in\{0,1\}$ and $s_{i}^{-} \in\{0,1\}$, with switching period $T_{\mathrm{sw}}$, to control transistors inside each $\mathrm{PU}_{i}$ as shown in Fig. 2. From voltage control viewpoint, the variables $u_{i}^{+}$and $u_{i}^{-}$can be viewed as control knobs to generate $v_{L i} \geq 0$ and $v_{L i} \leq 0$ respectively. Therefore, the positive control vector $u^{+}=\left[\begin{array}{lll}u_{1}^{+} & \cdots & u_{n}^{+}\end{array}\right]^{\mathrm{T}} \in$ $\mathcal{U}_{p} \subseteq \mathbb{R}_{+}^{n}$ generates positive $v_{L}$ with each $v_{L i} \geq 0$ and the 


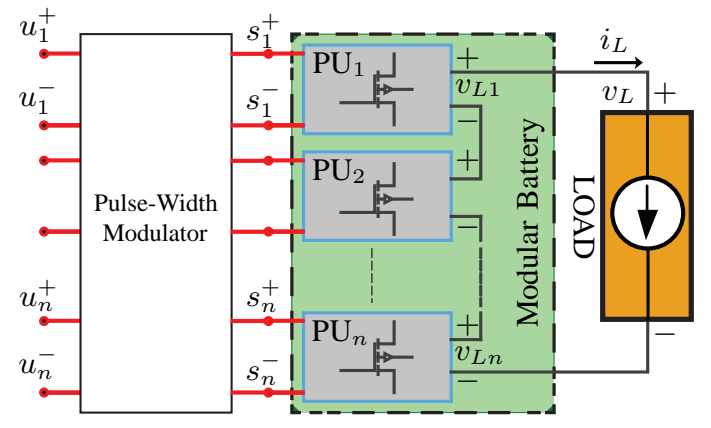

(a) Modular Battery with $n$ modules denoted by $\mathrm{PU}_{i}$.

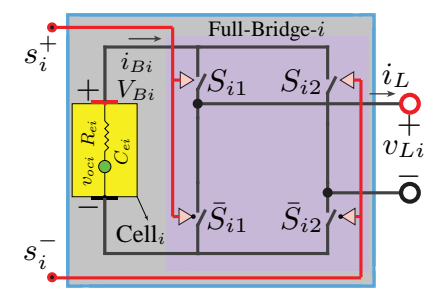

(b) FB-based $\mathrm{PU}_{i}$.

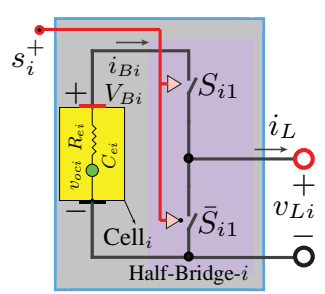

(c) HB-based $\mathrm{PU}_{i}$.
Fig. 2. Modular battery (inside green box) along with two alternative module topologies shown in figures 2(b) and 2(c).

negative control vector $u^{-}=\left[\begin{array}{lll}u_{1}^{-} & \cdots & u_{n}^{-}\end{array}\right]^{\mathrm{T}} \in \mathcal{U}_{n} \subseteq \mathbb{R}_{+}^{n}$ generates negative $v_{L}$ with each $v_{L i} \leq 0$. The full control

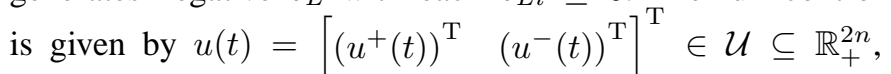
which gives the possibility of two control modes of the modular battery. Before defining these modes, three termspositive cell actuation, negative cell actuation, and bipolar cell actuation - are specified that are used frequently in this paper. It is positive actuation of $\mathrm{Cell}_{i}$ if only $u_{i}^{+}$is active $\left(u_{i}^{+} \neq 0\right)$, negative actuation of Cell ${ }_{i}$ if only $u_{i}^{-}$is active, and bipolar actuation if both $u_{i}^{+}$and $u_{i}^{-}$are simultaneously active subject to some assumptions (discussed below) about subsequent PWM generation method. Now two battery control modes are defined based on how $u^{+}$and $u^{-}$are employed.

Definition III.1 (Unipolar and Bipolar Control Modes). In unipolar control (UPC) mode, depending on the sign of demanded load voltage $v_{L d}$, either $u^{+}$is active (positive actuation of all cells) or $u^{-}$is active (negative actuation of all cells). Since $v_{L d}$ is always non-negative for $\mathrm{xEVs}$, only positive actuation of cells is considered under UPC mode here. This simpler mode does not allow polarity inversion of any cell in the string (i.e. $v_{L i}(t) v_{L j}(t) \geq 0$ ) during any switching cycle. This implies that at any time, either all cells are charging or all are discharging depending on the direction of $i_{L}$. In the bipolar control (BPC) mode, both $u^{+}$and $u^{-}$ may be simultaneously active (i.e. bipolar cell actuation). The BPC mode allows polarity inversion (i.e. $v_{L i}(t) v_{L j}(t) \leq 0$ ) of some cells in the string during each switching cycle. This simply implies that it is possible to charge some cells while discharging others at any time.

Note that the BPC mode, with two control variables per cell, improves the controllability properties of the modular battery system, which may make it easier to achieve the control objectives. However, it may require larger surplus voltage in the modular battery compared to that for UPC and may also generate extra battery losses due to negative cell actuation. In addition, the BPC mode also poses some modeling challenges (i.e. non-convexity may arise, see Remark 2), which need a special consideration regarding pulse placement method for PWM signal generation, see condition (1) below.

\section{B. Power Unit Architecture}

There are various dc/dc converter topologies that can be used inside PUs. Two particular architectures of $\mathrm{PU}_{i}$, based on full-bridge (FB) and half-bridge (HB) converters, considered in this study are shown in figures 2(b) and 2(c). The FBbased $\mathrm{PU}_{i}$, consisting of four bidirectional switches, can be operated in all four quadrants of the $i_{L}-v_{L i}$ plane using two unipolar switching functions $s_{i}^{+}(t)$ and $s_{i}^{-}(t)$. This makes it possible to voluntarily charge as well as discharge the battery module i.e. bidirectional battery power control. The HB-based $\mathrm{PU}_{i}$, on the other hand, can be operated in only $1^{\text {st }}$ and $2^{\text {nd }}$ quadrants of the $i_{L}-v_{L i}$ plane using $s_{i}^{+}(t)$. The control in the $2^{\text {nd }}$ quadrant is only possible during regeneration or external charging phases. Note that if FB-based $\mathrm{PU}_{i}$ is operated using UPC $\left(s_{i}^{-}(t)=0\right)$ then switch $\bar{S}_{i 2}$ is turned ON permanently. This implies that the switch $\bar{S}_{i 2}$ can be replaced with a short-circuit, which reduces FB-based $\mathrm{PU}_{i}$ to HB-based $\mathrm{PU}_{i}$. Therefore, both topologies are equivalent under UPC mode.

\section{Power Unit (or Cell) Switched Behavior}

There are three (two) different operationalmodes/switching-states of each FB-based $\mathrm{PU}_{i}$ (HB-based $\mathrm{PU}_{i}$ ). In Mode-1 $v_{L i}>0$, in Mode-2 $v_{L i}<0$ and in Mode-3 $v_{L i}=0$. These modes can be modeled using two unipolar switching functions $s_{i}^{+}$and $s_{i}^{-}$. For modeling convenience, this study assumes $s_{i}^{+}$and $s_{i}^{-}$to be orthogonal (non-overlapping) i.e.,

$$
\int_{t-T_{\mathrm{sw}}}^{t} s_{i}^{+}(\tau) s_{i}^{-}(\tau) \mathrm{d} \tau=0,
$$

where $T_{\mathrm{sw}}$ is the switching period of $s_{i}^{+}$and $s_{i}^{-}$. This orthogonality condition simply implies that $s_{i}^{+}$and $s_{i}^{-}$cannot be high simultaneously. Now using this condition, a single three-level bipolar PWM function $s_{i}(t)$ modeling three aforementioned modes is given by

$$
s_{i}(t)=s_{i}^{+}(t)-s_{i}^{-}(t)= \begin{cases}1, & \text { Mode-1 } \\ -1, & \text { Mode-2 } \\ 0, & \text { Mode-3 }\end{cases}
$$

Note that according to the condition (1), $s_{i}=0$ is generated using only $s_{i}^{+}=s_{i}^{-}=0$ i.e. by turning ON the lower transistors $\left(\bar{S}_{i 1}\right.$ and $\left.\bar{S}_{i 2}\right)$ and not the upper ones. Also note that only Modes 1 and 3 are available for HB-based $\mathrm{PU}_{i}$.

The signals $\left(i_{B i}, V_{B i}, i_{L}, v_{L i}\right)$ on two ports of each $\mathrm{PU}_{i}$ are linearly related through $s_{i}(t)$ as follows. The switched current through each $\mathrm{Cell}_{i}$ for a given load current $i_{L}$ is given by

$$
i_{B i}(t)=i_{L}(t) s_{i}(t)
$$


The switched terminal voltage of each $\mathrm{PU}_{i}$ is given by

$$
v_{L i}(t)= \begin{cases}d_{v i}^{+}(t), & s_{i}(t)=1 \\ 0, & s_{i}(t)=0 \\ -d_{v i}^{-}(t), & s_{i}(t)=-1\end{cases}
$$

where

$$
d_{v i}^{+}(t)=v_{o c i}-i_{L}(t) R_{e i}, \quad d_{v i}^{-}(t)=v_{o c i}+i_{L}(t) R_{e i},
$$

are cell terminal voltages, $V_{B i}(t)$, during discharging and charging respectively for $i_{L}>0$ where $v_{o c i}$ and $R_{e i}$ denote cell OCV and resistance. Based on orthogonality condition (1), the piecewise linear function (4) is equivalently represented by

$$
v_{L i}(t)=d_{v i}^{+}(t) s_{i}^{+}(t)-d_{v i}^{-}(t) s_{i}^{-}(t) .
$$

Now the variables $d_{v i}^{+}$and $d_{v i}^{-}$can also be interpreted as terminal voltages of Cell ${ }_{i}$ during its positive and negative actuation respectively. The terminal voltage and power of the modular battery are given by $v_{L}=\sum_{i=1}^{n} v_{L i}$ and $P_{L}=\sum_{i=1}^{n} P_{L i}$, where $P_{L i}=v_{L i} i_{L}$ is the terminal power of each $\mathrm{PU}_{i}$.

\section{Cell Averaging}

This study focuses on controlling the average behavior of the switched modular battery during each switching period $T_{\mathrm{Sw}}$ of $s_{i}(t)$ under both UPC and BPC modes. For this purpose, averaging of cell variables is done in this section in a setting, which is applicable to both UPC and BPC ${ }^{1}$. Two assumptions are employed: 1) the orthogonality condition (1) is satisfied and 2) $i_{L}(t)$ is constant during each cycle of a high-frequency PWM $s_{i}(t)$.

\section{A. Positive and Negative Controls (Duty Cycles)}

Assuming the orthogonality condition (1) is satisfied, the positive and negative controls (or duty cycles) of Cell ${ }_{i}$ during switching period $\left[t-T_{\mathrm{sw}}, t\right]$ are defined by

$$
\begin{aligned}
& u_{i}^{+}(t):=\frac{1}{T_{\mathrm{sw}}} \int_{t-T_{\mathrm{sw}}}^{t} s_{i}^{+}(\tau) \mathrm{d} \tau=\frac{T_{i}^{+}(t)}{T_{\mathrm{sw}}}, \\
& u_{i}^{-}(t):=\frac{1}{T_{\mathrm{sw}}} \int_{t-T_{\mathrm{sw}}}^{t} s_{i}^{-}(\tau) \mathrm{d} \tau=\frac{T_{i}^{-}(t)}{T_{\mathrm{sw}}},
\end{aligned}
$$

where $T_{i}^{+}(t)$ and $T_{i}^{-}(t)$ are ON time intervals of $s_{i}^{+}(t)$ and $s_{i}^{-}(t)$ respectively during switching period $\left[t-T_{\mathrm{sw}}, t\right]$. Note that the duty cycles can only be chosen such that $u_{i}^{+} \in[0,1], u_{i}^{-} \in[0,1]$, and $u_{i}^{+}+u_{i}^{-} \in[0,1]$. These constraints can be represented as a polytope

$$
\mathcal{U}_{i}=\left\{\left(u_{i}^{+}, u_{i}^{-}\right) \mid H_{u i} u_{i} \leq h_{u i}\right\}
$$

for suitably defined constraint matrix $H_{u i}$ and vector $h_{u i}$, where $u_{i}=\left[\begin{array}{ll}u_{i}^{+}(t) & u_{i}^{-}(t)\end{array}\right]^{\mathrm{T}}$. The set $\mathcal{U}_{i}$ is shown in Fig. 3(a) for UPC (using $u_{i}^{-}=0$ in (9)) and in Fig. 3(d) for BPC.

\footnotetext{
${ }^{1}$ In [12]-[14], the averaging was carried out assuming UPC mode.
}

\section{B. SOC and Temperature Controls}

Using $u_{i}^{+}$and $u_{i}^{-}$, two new control variables are defined as follows

$$
\begin{aligned}
& u_{g i}(t)=u_{i}^{+}(t)-u_{i}^{-}(t), \\
& u_{\ell i}(t)=u_{i}^{+}(t)+u_{i}^{-}(t) .
\end{aligned}
$$

The variables $u_{g i}$ and $u_{\ell i}$ respectively control average and rms currents in Cell $_{i}$ during each switching period [see next subsection]. Since the average and rms cell currents govern SOC and temperature dynamics respectively [see averaged model (20a) and (20b)], $u_{g i}$ and $u_{\ell i}$ are so-called SOC and temperature controls. The set of admissible SOC and temperature control actions can be represented by the following electro-thermal control polytope

$$
\mathcal{U}_{i}^{g \ell}=\left\{\left(u_{g i}, u_{\ell i}\right) \mid H_{u g \ell, i} u_{g \ell, i} \leq h_{u g \ell, i}\right\},
$$

for suitably defined constraint matrix $H_{u g \ell, i}$ and vector $h_{u g \ell, i}$, where $u_{g \ell, i}=\left[\begin{array}{ll}u_{g i}(t) & u_{\ell i}(t)\end{array}\right]^{\mathrm{T}}$. The set $\mathcal{U}_{i}^{g \ell}$ is shown in Fig. 3(b) for UPC and in Fig. 3(e) for BPC.

\section{Average and RMS Currents}

Using definitions (2), (7), (8), and relation (3), average and rms cell currents during each switching period can be computed as follows. The average current of $\mathrm{Cell}_{i}$ is given by

$$
\begin{aligned}
i_{B a i}(t) & =\frac{1}{T_{\mathrm{sw}}} \int_{t-T_{\mathrm{sw}}}^{t} i_{B i}(\tau) \mathrm{d} \tau \\
& =i_{L}(t)\left[u_{i}^{+}(t)-u_{i}^{-}(t)\right]=i_{L}(t) u_{g i}(t)
\end{aligned}
$$

Similarly, the rms current of $\mathrm{Cell}_{i}$ is defined by

$$
i_{B r i}^{2}(t)=\frac{1}{T_{\mathrm{sw}}} \int_{t-T_{\mathrm{sw}}}^{t} i_{B i}^{2}(\tau) \mathrm{d} \tau=\frac{i_{L}^{2}(t)}{T_{\mathrm{sw}}} \int_{t-T_{\mathrm{sw}}}^{t} s_{i}{ }^{2}(\tau) \mathrm{d} \tau,
$$

which, using (2) and orthogonality condition (1), is given by

$$
i_{B r i}^{2}(t)=i_{L}^{2}(t)\left[u_{i}^{+}(t)+u_{i}^{-}(t)\right]=i_{L}^{2}(t) u_{\ell i}(t) .
$$

Now, defining $i_{B a r, i}=\left[\begin{array}{ll}i_{B a i} & i_{B r i}^{2}\end{array}\right]^{\mathrm{T}}$, the set of admissible average and rms currents can be represented by a polytope

$$
\mathcal{I}_{i}^{a r}=\left\{\left(i_{B a i}, i_{B r i}^{2}\right) \mid H_{i B a r, i}(t) i_{B a r, i} \leq h_{i B a r, i}\right\},
$$

for suitably defined $H_{i B a r, i}$ and $h_{i B a r, i}$. The set is shown in figures 3(c) and 3(f) for UPC and BPC respectively.

Remark 1 (UPC and BPC Comparison based on $\mathcal{I}_{i}^{a r}$ ). Note that there is a linear relationship (one-to-one coupling) between average and rms cell currents under constant load for UPC mode, see line segments representing set of feasible average and rms cell currents in Fig. 3(c). For any constant load current, average and rms currents $\left(i_{B a i}\right.$ and $\left.i_{B r i}^{2}\right)$ of any $\mathrm{Cell}_{i}$ can be chosen only along a certain line. To change rms value of cell current without affecting its average value requires change in magnitude of load current. Similarly, to change cell average current without affecting the rms requires reversal in direction of load current. Therefore, load current variation, both in magnitude and direction, is favorable for achieving simultaneous thermal and SOC balancing using UPC mode otherwise it may be a daunting task under constant high load current. For BPC mode, on the other hand, average 


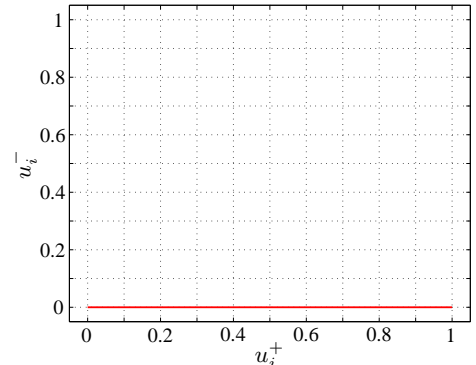

(a) $\mathcal{U}_{i}$ : Set of feasible duty cycles under UPC.

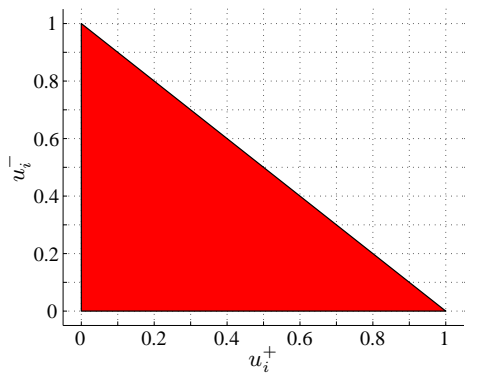

(d) $\mathcal{U}_{i}$ : Set of feasible duty cycles under BPC.

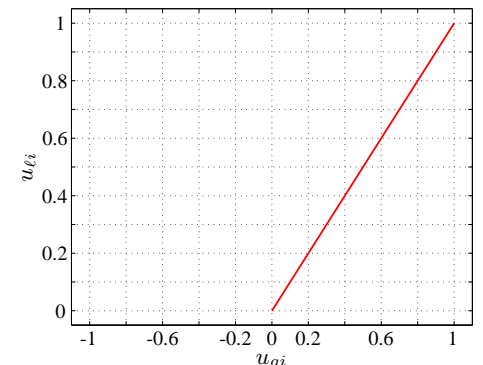

(b) $\mathcal{U}_{i}^{g \ell}:$ Set of feasible SOC and temperature controls under UPC.

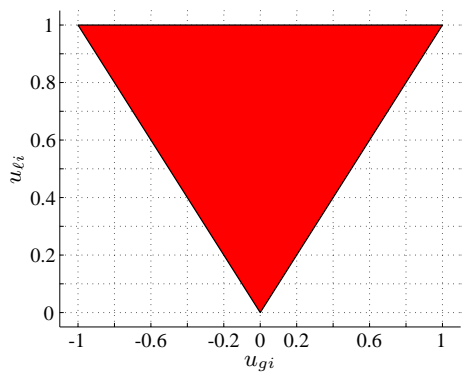

(e) $\mathcal{U}_{i}^{g \ell}:$ Set of feasible SOC and temperature controls under BPC.

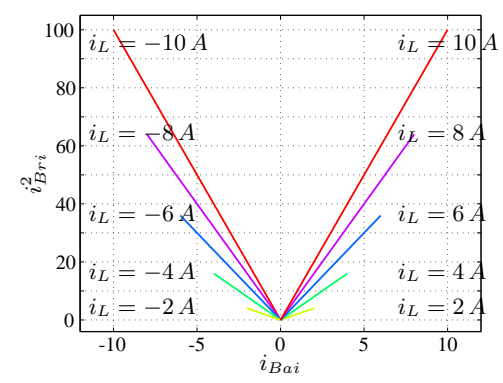

(c) $\mathcal{I}_{i}^{a r}(t)$ : Set of average and rms currents for given $i_{L}$ under UPC.

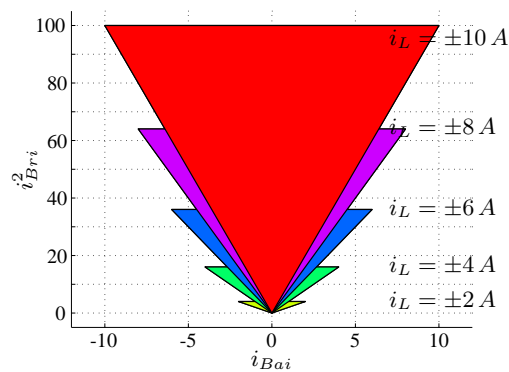

(f) $\mathcal{I}_{i}^{a r}(t)$ : Set of average and rms currents for given $i_{L}$ under BPC.

Fig. 3. Various constraint sets for $\mathrm{Cell}_{i}$, as defined in (9), (12), and (15), are shown for UPC and BPC modes in the first and second row respectively. See these definitions and Remark 1 for interpretation. Note that 3(c) and 3(f) can be obtained respectively by scaling 3(b) and 3(e) along the parabola $i_{L}^{2}$.

and rms cell currents are loosely coupled under constant loads, see triangular polytopes representing set of feasible average and rms cell currents in Fig. 3(f). This larger set gives a possibility of somewhat independent adjustment of $i_{B a i}$ and $i_{B r i}$, which is favorable for simultaneous thermal and SOC balancing. Therefore, variation in magnitude and direction of load current is not strictly needed for BPC.

From this simple reasoning, it can be readily seen that $B P C$ would result in tighter balancing subject to negative cell actuation $\left(u_{i}^{-}(t)>0\right)$, which is feasible if the voltage demand $v_{L d}(t)$ is sufficiently lower than the maximum voltage capacity $v_{L, \max }(t)$ [see equation (24) for definition] of the modular battery. This may require redundant modules in the battery pack.

\section{Average Voltage}

Using (6), the average terminal voltage of $\mathrm{PU}_{i}$ is given by

$$
\begin{aligned}
v_{L a i}(t) & =\frac{1}{T_{\mathrm{sw}}} \int_{t-T_{\mathrm{sw}}}^{t} v_{L i}(\tau) \mathrm{d} \tau \\
& =d_{v i}^{+}(t) u_{i}^{+}(t)-d_{v i}^{-}(t) u_{i}^{-}(t) .
\end{aligned}
$$

The terminal voltage of the modular battery is thus given by

$$
v_{L a}(t)=\sum_{i=1}^{n} v_{L a i}(t)=D_{v}^{+}(t) u^{+}(t)-D_{v}^{-}(t) u^{-}(t)
$$

where

$$
\begin{aligned}
& D_{v}^{+}(t)=\left[\begin{array}{lll}
d_{v 1}^{+}(t) & \cdots & d_{v n}^{+}(t)
\end{array}\right], \\
& D_{v}^{-}(t)=\left[\begin{array}{lll}
d_{v 1}^{-}(t) & \cdots & d_{v n}^{-}(t)
\end{array}\right],
\end{aligned}
$$

are vectors of terminal voltages of $n$ cells during discharging and charging respectively for $i_{L}>0$.

\section{E. Average Power}

The total terminal power of the modular battery is given by

$$
P_{L a}(t)=\sum_{i=1}^{n} P_{L a i}(t)=D_{p}^{+}(t) u^{+}(t)-D_{p}^{-}(t) u^{-}(t),
$$

where $P_{L a i}=v_{L a i} i_{L}$ is the average terminal power of each Cell $_{i}$ and $D_{p}^{+}=i_{L} D_{v}^{+}$and $D_{p}^{-}=i_{L} D_{v}^{-}$are vectors of cell terminal powers during discharging and charging respectively for $i_{L}>0$.

Remark 2. The use of two switching functions and orthogonality condition (1) has greatly simplified the derivation of averaged quantities (affine functions of duty cycles) for BPC here compared to approach in [12] that leads to non-convex terms like product of variables $\left(u_{i}^{+} \cdot u_{i}^{-}\right)$.

\section{Averaged State-Space Electro-thermal Model}

The averaged state-space electro-thermal model of an aircooled modular battery consisting of $n$ modules with ideal switches is presented on standard form here using averaged variables $i_{B a i}$ and $i_{B r i}^{2}$ [see (13) and (14)] as inputs for SOC and thermal dynamics respectively. The cell electrical dynamics is studied using a simple cell model (OCV-R), see [23]. The OCV of all cells is assumed constant in this study. This approximation is somewhat justified for certain types of lithium-ion cells (for example $\mathrm{LiFePO}_{4} /$ graphite (LFP)) if battery is operated in a typical SOC window of $20 \%$ to $90 \%$ [5]. The battery thermal dynamics is modeled using lumped capacitance and flow network modeling approach, which has been experimentally validated in [8] as well as in [24]-[26]. The model considers only cell casing temperature with constant coolant temperature and speed at inlet. 


\section{A. Model of One Module}

The averaged electro-thermal model of any module $\mathrm{PU}_{i}$ of the modular battery for a given load current $i_{L}(t)$ is given by

$$
\begin{aligned}
\dot{\xi}_{i}(t) & =-\frac{1}{3600 C_{e i}} i_{L}(t)\left(u_{i}^{+}(t)-u_{i}^{-}(t)\right), \\
\dot{T}_{s i}(t) & =\sum_{j=1}^{i} a_{t i j} T_{s j}+\frac{R_{e i}}{C_{s i}} i_{L}^{2}\left(u_{i}^{+}+u_{i}^{-}\right)+w_{t i} T_{f 0}, \\
v_{L a i}(t) & =d_{v i}^{+}(t) u_{i}^{+}(t)-d_{v i}^{-}(t) u_{i}^{-}(t),
\end{aligned}
$$

where temperature, $T_{s i}$, and SOC, $\xi_{i}$, are states, $T_{f 0}$ is the constant inlet coolant temperature (measured disturbance), $v_{L a i}$ is the terminal voltage of $\mathrm{PU}_{i}, u_{i}^{+}$and $u_{i}^{-}$are control variables defined in (7) and (8), and $d_{v i}^{+}$and $d_{v i}^{-}$are defined in (5). The cell parameters $R_{e i}, C_{e i}$, and $C_{s i}$ are the internal resistance, the coulomb capacity, and the heat capacity of $\mathrm{Cell}_{i}$. The coefficient $a_{t i j}$ describes unidirectional thermal coupling from upstream $\mathrm{Cell}_{j}$ to downstream $\mathrm{Cell}_{i}$ due to convective heat transfer, whereas the coefficient $w_{t i}=-\sum_{j=1}^{i} a_{t i j}$ describes the influence of $T_{f 0}$ on $\mathrm{Cell}_{i}$, see [12], [16], [17] for detailed derivation and definition of these coefficients.

\section{B. Complete Model}

Using (20a)-(20c) as basic building block and treating $T_{f 0}$ as a dummy state, the averaged electro-thermal model of a complete $n$-cell modular battery is given by the following standard linear time-varying state-space system

$$
\begin{aligned}
& \dot{x}(t)=A x(t)+B\left(i_{L}(t)\right) u(t), \\
& y(t)=C x(t)+D\left(i_{L}(t)\right) u(t) .
\end{aligned}
$$

Here $x(t)=\left[\begin{array}{lll}\xi^{\mathrm{T}}(t) & \vartheta^{\mathrm{T}}(t)\end{array}\right]^{\mathrm{T}} \in \mathbb{R}^{2 n+1}$ is the full state vector, $\xi(t)=\left[\begin{array}{lll}\xi_{1}(t) & \cdots & \xi_{n}(t)\end{array}\right]^{\mathrm{T}} \in \mathbb{R}^{n}$ is a vector of SOCs, $\vartheta(t)=\left[\begin{array}{ll}T_{s}^{\mathrm{T}}(t) & T_{f 0}\end{array}\right]^{\mathrm{T}} \in \mathbb{R}^{n+1}$ is an augmented thermal state with $T_{s}(t)=\left[\begin{array}{lll}T_{s 1}(t) & \cdots & T_{s n}(t)\end{array}\right]^{\mathrm{T}} \in \mathbb{R}^{n}$, $u(t)=\left[\begin{array}{ll}\left(u^{+}(t)\right)^{\mathrm{T}} & \left(u^{-}(t)\right)^{\mathrm{T}}\end{array}\right]^{\mathrm{T}} \in \mathbb{R}^{2 n}$ is the control vector, $y(t)=\left[\begin{array}{ll}\vartheta^{\mathrm{T}}(t) & v_{L a}(t)\end{array}\right]^{\mathrm{T}} \in \mathbb{R}^{n+2}$ is the output vector, and

$$
v_{L a}(t)=D_{v}(t) u(t),
$$

is the battery terminal voltage. All the state-space matrices $\left(A, B, C, D, D_{v}\right)$ are defined in the Appendix. The discretetime state-space model is given by

$$
\begin{aligned}
x(k+1) & =A_{d} x(k)+B_{d}\left(i_{L}(k)\right) u(k), \\
y(k) & =C x(k)+D\left(i_{L}(k)\right) u(k),
\end{aligned}
$$

where $A_{d}$ and $B_{d}(k)$ are obtained using Euler approximation of (21a) assuming $i_{L}$ to be constant during each sampling interval $[k h,(k+1) h]$ where $h$ is a sampling step size.

\section{Voltage Capacity/Limit}

The modular battery voltage is limited to an interval $v_{L a}(k) \in\left[v_{L, \min }(k), v_{L, \max }(k)\right]$ where

$$
v_{L, \min }(k)=-D_{v}^{-}(k) \cdot 1_{n}, \quad v_{L, \max }(k)=D_{v}^{+}(k) \cdot 1_{n},
$$

are so-called minimum and maximum voltage capacities of the modular battery at any time instant for any $i_{L}(k)>0$.

\section{Control Constraint/Limit}

The constraint set for the $n$-cell modular battery is given by

$$
\mathcal{U}=\prod_{i=1}^{n} \mathcal{U}_{i}=\left\{u \mid H_{u} u \leq h_{u},\right\}
$$

for suitably defined $H_{u}$ and $h_{u}$ where $\mathcal{U}_{i}$ is the control constraint set for $\mathrm{Cell}_{i}$ as defined in (9).

\section{Control Problem Formulation}

\section{A. Preliminaries}

Let us define SOC and temperature error vectors

$$
\begin{aligned}
e_{\xi}(k) & =\xi(k)-\bar{\xi}(k) \cdot 1_{n}=M_{e} \xi(k), \\
e_{T_{s}}(k) & =T_{s}(k)-\bar{T}_{s}(k) \cdot 1_{n}=M_{e} T_{s}(k),
\end{aligned}
$$

where $\bar{\xi}(k)=\frac{1}{n} 1_{n}^{\mathrm{T}} \xi(k)$ and $\bar{T}_{s}(k)=\frac{1}{n} 1_{n}^{\mathrm{T}} T_{s}(k)$ are instantaneous mean SOC and mean temperature of the modular battery and can be considered as reference signals here. The matrix

$$
M_{e}=\left(I_{n}-\frac{1}{n} 1_{n \times n}\right) \in \mathbb{R}^{n \times n},
$$

maps each state vector to its corresponding error vector. The control objective is to minimize these errors (simultaneous thermal and SOC balancing) and reduce mean battery temperature while regulating the battery terminal voltage at the demand setpoint (i.e., $v_{L a}=v_{L d}$ ) using $u \in \mathcal{U}$.

\section{B. Control Method: Overview}

If complete future load demand is available then full optimal control trajectory can be generated to achieve the control objectives by solving off-line a state and control constrained convex optimization problem over whole driving horizon [12][14]. However, this assumption is quite unrealistic especially in xEVs. Therefore, a one-step LQ MPC based method for UPC mode is proposed in [16], [17] to solve the problem without using any future driving information. The proposed method prioritizes the load voltage regulation, whereas thermal and SOC balancing are achieved as secondary objectives by optimally using any redundancy available in the modular battery. The control strategy is based on the decomposition of total controller into two orthogonal components as follows

$$
u(k)=\left(u_{v}(k)+u_{b}(k)\right) \in \mathcal{U},
$$

where control $u_{v}(k) \in \mathcal{N}\left(D_{v}(k)\right)^{\perp}$ is for voltage control and $u_{b}(k) \in \mathcal{N}\left(D_{v}(k)\right)$ is for balancing control where $\mathcal{N}\left(D_{v}(k)\right)$ is the nullspace of $D_{v}(k)$ and $\mathcal{N}\left(D_{v}(k)\right)^{\perp}$ is its orthogonal complement. The time-varying nullspace of $D_{v}(k)$ is a hyperplane in $\mathbb{R}^{m}$ given by

$$
\mathcal{N}\left(D_{v}\right)=\left\{u(k) \mid D_{v}(k) u(k)=0\right\}=\mathcal{R}\left(V_{\mathrm{n}}\right) \subseteq \mathbb{R}^{m},
$$

where $m$ is the number of control variables and $\mathcal{R}\left(V_{\mathrm{n}}\right)$ is the range-space of nullspace basis matrix

$$
V_{\mathrm{n}}(k)=\left[\begin{array}{lll}
v_{\mathrm{n}, 1}(k) & \cdots & v_{\mathrm{n}, m-1}(k)
\end{array}\right] \in \mathbb{R}^{m \times m-1},
$$

containing parameterized basis vectors $v_{\mathrm{n}, i} \in \mathbb{R}^{m}$ where the subscript ' $\mathrm{n}$ ' stands for nullspace. The proposed orthogonal decomposition guarantees the voltage constraint satisfaction 
while giving the possibility of simultaneous thermal and SOC balancing. The voltage control problem is a minimum norm problem, whereas the balancing problem is formulated as a control constrained LQ MPC problem.

In this paper, a similar control structure as summarized above is employed, but it is tailored towards the BPC mode. A particular choice of $V_{\mathrm{n}}$ with $m=2 n$, obtained using MATLAB ${ }^{\circledR}$ Symbolic Toolbox, is given by

$$
V_{\mathrm{n}}(k)=\left[\begin{array}{c}
V_{\mathrm{n}}^{\prime}(k) \\
I_{2 n-1}
\end{array}\right] \in \mathbb{R}^{2 n \times 2 n-1},
$$

where $V_{\mathrm{n}}^{\prime}(k)=\left[\begin{array}{ll}\frac{-D_{v}^{+}(2: n)}{d_{v 1}^{+}} & \frac{D_{v}^{-}}{d_{v 1}^{+}}\end{array}\right] \in \mathbb{R}^{1 \times(2 n-1)}$ and $D_{v}^{+}(2: n)$ (indexed using Matlab notation) is a row vector with last $n-1$ elements of $D_{v}^{+}$. The formulation of voltage and balancing control problems for BPC mode is given below, and UPC is treated as a special case of BPC.

\section{Voltage Controller: Minimum Norm Problem}

The control $u_{v}$ at each time instant can be computed by directly solving the output equation (22) to satisfy $v_{L a}=v_{L d}$ for any given $i_{L}$. However, $D_{v} u_{v}=v_{L d}$ has infinite solutions due to nonempty nullspace of $D_{v}$ that provides $2 n-1$ degreesof-freedom to generate $v_{L a}$. A unique solution $u_{v} \in \mathcal{N}\left(D_{v}\right)^{\perp}$ is given by the following least norm problem

$$
\begin{array}{ll}
\text { minimize } & \left\|u_{v}(k)\right\|^{2} \\
\text { subject to } & D_{v}(k) u_{v}(k)=v_{L d}(k), \\
& u_{v}(k) \in \mathcal{U},
\end{array}
$$

which is feasible for load demands $i_{L}(k) \in\left[i_{L, \min }, i_{L, \max }\right]$ and $v_{L d}(k) \in\left[0, v_{L d, \max }\right]$ with appropriately defined limits $i_{L, \min }, i_{L, \max }$, and $v_{L d, \max }<v_{L, \max }(k)$. The problem (P-I) has an analytical solution as motivated below.

The equality constraint in (P-I) can be represented by

$$
v_{L d}=D_{v}(k) u_{v}(k)=D_{v}^{+}(k) u_{v}^{+}(k)-D_{v}^{-}(k) u_{v}^{-}(k)
$$

where $D_{v}^{+}(k) \geq 0$ and $D_{v}^{-}(k) \geq 0$ are defined in (18a) and (18b) respectively. Since increasing $u_{v}^{-}$always decreases the terminal voltage $v_{L a}$ for any given $u_{v}^{+}$, it is not optimal to use $u_{v}^{-}$to generate voltage $v_{L a}$ as it increases the length (i.e., norm) of vector $u_{v}$. Therefore, the optimizer must set

$$
u_{v}^{-}=0
$$

to minimize the norm ${ }^{2}$ of $u_{v}$. Therefore, the optimization problem (P-I) is equivalent to

$$
\begin{array}{ll}
\operatorname{minimize} & \left\|u_{v}^{+}(k)\right\| \\
\text { subject to } & D_{v}^{+}(k) u_{v}^{+}(k)=v_{L d}(k), \\
& u_{v}^{+}(k) \in \mathcal{U}_{p},
\end{array}
$$

which is simpler than the problem (P-I) and has an analytical solution given by [16]

$$
u_{v}^{+}(k)=\left(D_{v}^{+}(k)\right)^{\dagger} v_{L d}(k)
$$

\footnotetext{
${ }^{2}$ This claim, shown here based on a simple argument, can also be proved formally using KKT conditions from mathematical optimization theory.
}

where $\left(D_{v}^{+}\right)^{\dagger}=D_{v}^{+^{\mathrm{T}}}\left(D_{v}^{+} D_{v}^{+^{\mathrm{T}}}\right)^{-1}$ is a right pseduo-inverse of $D_{v}^{+}$. The complete solution is given by

$$
u_{v}(k)=\left[\begin{array}{c}
u_{v}^{+}(k) \\
u_{v}^{-}(k)
\end{array}\right]=\left[\begin{array}{c}
\left(D_{v}^{+}(k)\right)^{\dagger} \\
0_{n}
\end{array}\right] v_{L d}(k) .
$$

The above solution $u_{v}(k) \in \mathcal{R}\left(D_{v}(k)^{\mathrm{T}}\right)$ is guaranteed to be inside $\mathcal{U}$ at any time instant if $i_{L}(k) \in\left[i_{L, \min }, i_{L, \max }\right]$ and

$$
v_{L d, \max }<v_{L, \max }(k), \quad \forall k,
$$

see [16] for the proof of this claim. Note that $u_{v}$ is a feedforward control, which is computed based on the load demand $v_{L d}$ and $i_{L}$ at each time instant.

\section{Balancing Controller: Constrained LQ MPC}

The balancing objectives can be achieved by appropriately choosing $u_{b}(k) \in \mathcal{U}_{b}(k) \subseteq \mathcal{N}\left(D_{v}\right)$ where $\mathcal{U}_{b}(k)$, defined in (38), is a time-varying set of feasible balancing controls. The balancing control can be represented by the linear combination of the basis vectors of nullspace as follows

$$
u_{b}(k)=\sum_{i=1}^{2 n-1} \rho_{b i}(k) v_{\mathrm{n}, i}(k)=V_{\mathrm{n}}(k) \rho_{b}(k) \in \mathcal{U}_{b}(k),
$$

where $V_{\mathrm{n}}$ is given by (31) and $\rho_{b} \in \mathbb{R}^{2 n-1}$ are coefficients of null-space basis vectors. These coefficients are computed by solving a constrained LQ problem in a receding horizon fashion. The problem formulation is given below.

1) Balancing Control Constraint Polytope: From (25), (29), and (30), the balancing control polytope is defined as follows

$$
\mathcal{U}_{b}(k)=\left\{u_{b} \mid H_{u_{b}} u_{b} \leq b_{u_{b}}, D_{v} u_{b}=0\right\} \subseteq \mathcal{N}\left(D_{v}\right),
$$

which is a so-called truncated nullspace where

$$
H_{u_{b}}=H_{u}, \quad b_{u_{b}}(k)=h_{u}-H_{u} u_{v}(k),
$$

are time-varying inequality constraint matrices. In simple words, choosing $u_{b} \in \mathcal{U}_{b}$ guarantees $u \in \mathcal{U}$ at each time instant without violating voltage constraint.

2) Balancing Objective Function: The standard one-step quadratic function given by

$$
J\left(x(k), \rho_{b}(k)\right)=\left[\|x(k+1)\|_{\bar{P}_{x}}^{2}+\left\|\rho_{b}(k)\right\|_{R_{\rho_{b}}}^{2}\right],
$$

with state penalty weighting matrix

$$
\bar{P}_{x}=\operatorname{blkdiag}\left(\gamma_{1} M_{e}^{\mathrm{T}} P_{E} M_{e}, \gamma_{2} M_{e}^{\mathrm{T}} P_{T} M_{e}+\gamma_{3} \frac{p_{\bar{t}}}{n^{2}} 1_{n \times n}, 0\right),
$$

encodes balancing objectives by adding cost for increase in balancing errors and mean battery temperature. The matrix $R_{\rho_{b}}(k)=\gamma_{4} V_{n}^{\mathrm{T}}(k) R_{u_{b}} V_{\mathrm{n}}(k)$ with $R_{u_{b}}=$ $\operatorname{blkdiag}\left(R_{u_{b}^{+}}, R_{u_{b}^{-}}\right)$is a penalty weight for $\rho_{b}$ where $R_{u_{b}^{+}}$ and $R_{u_{b}^{-}}$are penalties on $u_{b}^{+}$and $u_{b}^{-}$(positive and negative balancing controls) respectively. Note that $R_{u_{b}^{-}} \gg R_{u_{b}^{+}}$is used to reduce subsequent extra losses due to negative cell actuation. 
3) Constrained LQ MPC Standard Form: Now using (40), the balancing control problem can be easily formulated on the following standard control-constrained $L Q$ form, which is solved to find the balancing control decision $u_{b}(k)$ at each time step $k \in\left\{0, \cdots, N_{d}-1\right\}$ in the one-step MPC framework.

$$
\begin{array}{cl}
\operatorname{minimize} & J\left(x(k), \rho_{b}(k)\right) \\
\text { subject to } & x(k+1)=A_{d} x(k)+\bar{B}_{d}(k) \rho_{b}(k), \\
& u_{b}(k)=V_{\mathrm{n}}\left(i_{L}(k)\right) \rho_{b}(k) \in \mathcal{U}_{b}(k),
\end{array}
$$

with optimization variables $x(k+1)$ and $\rho_{b}(k)$ for a given initial state $x(k)$ where $N_{d}$ is the driving horizon and $\mathcal{U}_{b}(k)$ is defined in (38). Note that, by substituting $u(k)$ with $u_{b}(k)=$ $V_{\mathrm{n}}(k) \rho_{b}(k)$ as a control variable in (23a), the system dynamics is obtained in terms of new control variable $\rho_{b}(k)$ as shown in (P-IV) above where $\bar{B}_{d}(k)=B_{d}(k) \cdot V_{\mathrm{n}}(k)$. The voltage control $u_{v}(k)$ needed for solving the problem (P-IV) is already computed whereas the load current demand $i_{L}(k)$ is assumed to be perfectly known at each time step. The proposed control method is summarized as Algorithm 1 where the UPC mode becomes a special case of BPC by presetting $u^{-}=0$. The block diagram of the complete battery control system is shown in Fig. 4.

Remark 3. The cell resistance varies slightly as a function of temperature in normal operating range $[25,40]{ }^{\circ} \mathrm{C}$. In addition, it is also likely to have model mismatch (parametric uncertainty). However, it is shown in our earlier study [16] for UPC mode that the small resistance variation and parametric uncertainty have no significant effect on control performance. Therefore, cell resistance is assumed to be constant for control design in this paper as well. The resistance variation over large temperature range can be compensated using gainscheduling at much slower rate.
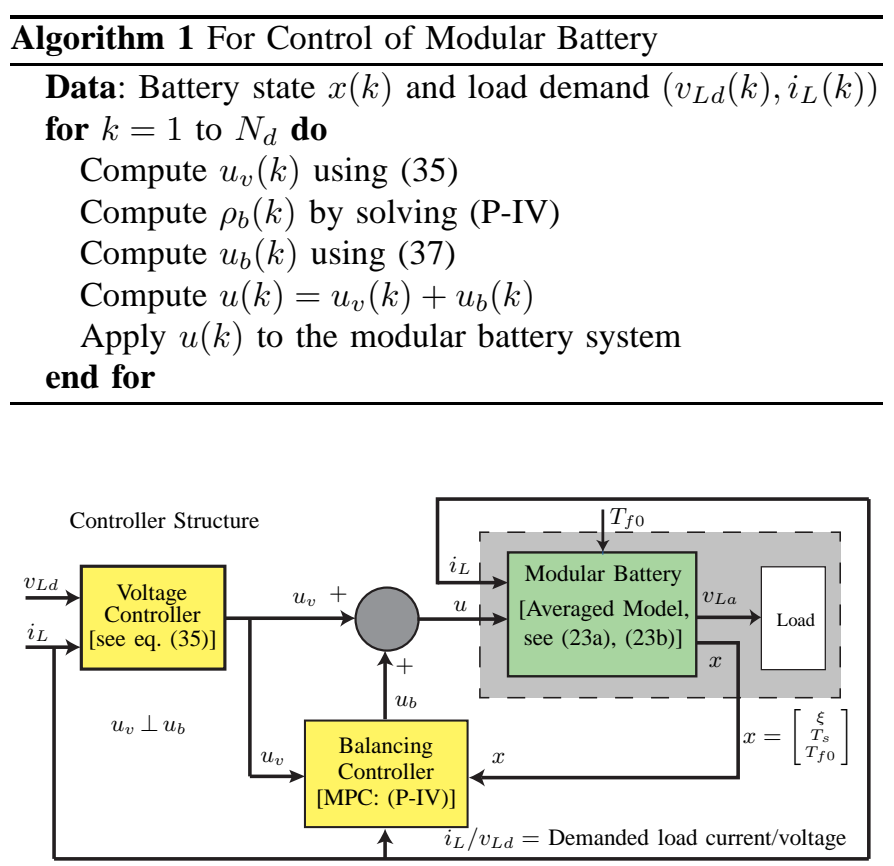

Fig. 4. Block diagram of closed-loop control system of the modular battery.

\section{Simulation Setup}

\section{A. Battery Configuration and Load Profile}

The modular battery control system of Fig. 4 has been simulated for 4 modules, each containing one cell $(3.3 \mathrm{~V}$, $2.3 A h$, A123 ANR26650M1A). The nominal values of cell's electro-thermal parameters, shown in Table I, have been taken from [24]-[26]. The actual cells are assumed to have capacity, SOC, and resistance distribution as shown in Fig. 5. In this parametric distribution, cells 3 and 4 have higher resistance as well as higher initial dischargeable capacity than the other cells. This implies conflicting cell usage requirements for SOC and thermal balancing during discharging, which makes the control task more challenging. Therefore, it is interesting to evaluate the balancing performance under this parametric variation for various real world and certification drive cycles. In particular, results are presented for USO6 drive cycle, which is representative of high speed highway driving (aggressive driving behavior) and is challenging for achieving simultaneous thermal and SOC balancing [16]. In addition, constant high speed motorway driving is also considered for thorough evaluation of balancing performance under most unfavorable condition i.e. little load current variation during driving. For thorough performance evaluation, two trips of each drive cycle are considered, where each trip is followed by the battery charging at constant $4 c$. The demanded battery load current $i_{L}$ (in $c$-rate) and its histogram for both drive cycles are shown in Fig. 6. The current data were obtained at $1 \mathrm{~Hz}$ by simulation of Toyota Prius PHEV in full EV mode in Advisor [27]. The demanded battery load voltage $v_{L d}$ is assumed as a constant dc-link voltage of a three-phase two-level inverter. It is chosen as $9.25 \mathrm{~V}$ to satisfy condition (36), at each time instant of both drive cycles, for the 4 cell modular battery considered here.

TABLE I

Simulation Setup: Cell Parameters and Controller Setting

\begin{tabular}{l|l|l|l}
\hline Parameter & Symbol & Value & Unit \\
\hline \multicolumn{3}{c}{ Cell Parameters } \\
\hline No. of Cells & $n$ & 4 & - \\
Nominal OCV & $v_{o c i}^{\star}$ & 3.3 & $V$ \\
Nominal Resistance & $R_{e i}^{\star}$ & 11.4 & $m \Omega$ \\
Nominal Capacity & $C_{e i}^{\star}$ & 2.3 & $A h$ \\
Heat Capacity & $C_{s i}$ & $71.50, \forall i$ & $J K^{-1}$ \\
Thermal Resistance & $R_{u i}$ & $3.03, \forall i$ & $K W^{-1}$ \\
Air Flow Rate & $\dot{V}_{f}$ & 0.0095 & $m^{3} s^{-1}$ \\
Air Thermal Conductance & $c_{f}$ & 11.1105 & $W K^{-1}$ \\
Inlet Fluid Temperature & $T_{f 0}$ & 25 & ${ }^{\circ} \mathrm{C}$ \\
Load Voltage Demand & $v_{L d}$ & 9.25 & $V$ \\
OCV Vector & $v_{o c}$ & $v_{o c i}^{\star} 1_{n}$ & $V$ \\
\hline \multicolumn{3}{c}{ Controller Setting } \\
\hline SOC Deviation Allowance & $\delta \xi$ & $2.5 \%$ & - \\
Temp. Deviation Allowance & $\delta T_{s}$ & 1 & ${ }^{\circ} \mathrm{C}$ \\
Control Sampling Interval & $h$ & 1 & $\mathrm{~s}$ \\
\hline
\end{tabular}

\section{B. Variable Definitions for Performance Comparison}

Some new variables are introduced to compare battery performance under UDO, UPC, and BPC modes in next section. To illustrate the balancing performance, variables $\left\|e_{\xi}(k)\right\|_{\infty}$ 


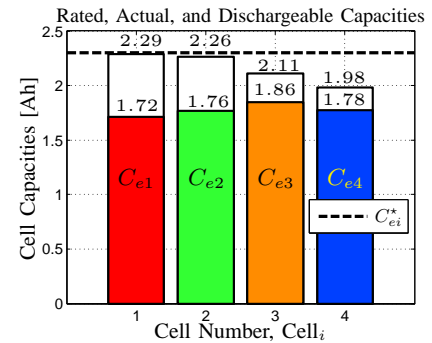

(a)

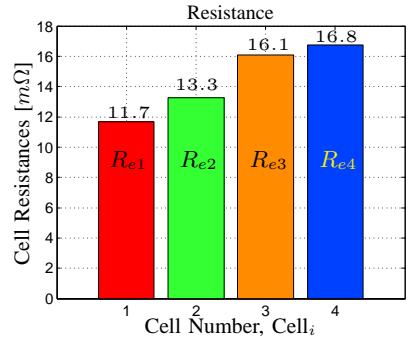

(b)
Fig. 5. (a) Capacity and (b) resistance distributions of cells. Fig. 5(a) shows actual capacity $\left(C_{e i}\right.$ i.e., container height $)$, dischargeable capacity $\left(C_{e d, i}=\right.$ $\xi_{i} C_{e i}$ i.e., filled container level), and nominal capacity, $C_{e i}^{\star}$, of cells.
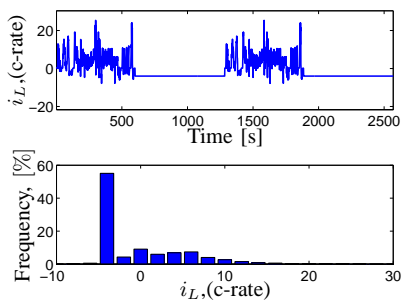

(a)
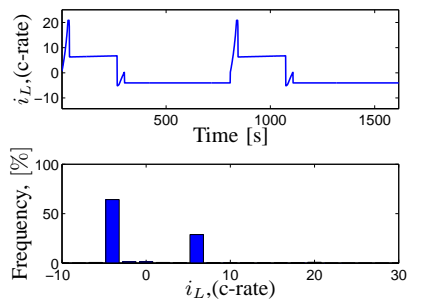

(b)
Fig. 6. Battery load current and the histogram for two trips of (a) US06 and (b) constant $80 \mathrm{mph}$ drive cycle along with $4 c$ charging after each trip.

and $\left\|e_{T_{s}}(k)\right\|_{\infty}$ [see (26) and (27) for definitions of $e_{\xi}$ and $e_{T_{s}}$ ] are used, which give the maximum SOC and temperature deviations (balancing errors) in the battery at any time instant. The comparison is also done in terms of effective battery capacity given by [5], [28]

$$
C_{\mathrm{B}}=\frac{1}{n} \sum_{i=1}^{n} C_{e i},
$$

for a battery pack with a lossless active balancing device as in UPC and BPC modes, whereas

$$
C_{\mathrm{B}}=\min _{i}\left(C_{e d, i}\right)+\min _{i}\left(C_{e c, i}\right),
$$

for a battery pack with cell imbalances as in UDO where $C_{e d, i}=\xi_{i} C_{e i}$ and $C_{e c, i}=\left(1-\xi_{i}\right) C_{e i}$ are dischargeable and chargeable cell capacities.

In addition to balancing performance, it is also important to compare battery losses. For this purpose, so-called local and mean efficiencies of battery pack are defined as follows

$$
\begin{aligned}
\eta_{B}(k) & = \begin{cases}\frac{P_{B}(k)}{P_{B g}(k)}, & i_{L}(k)>0 \\
\frac{P_{B g}(k)}{P_{B}(k)}, & i_{L}(k)<0\end{cases} \\
\bar{\eta}_{B} & =m_{\eta_{B}}
\end{aligned}
$$

where variables $P_{B}$ and $P_{B g}$ are defined in Table II and $m_{\eta_{B}}$ denotes mean of $\left\{\eta_{B}(k)\right\}_{k=1}^{N_{d}}$. Table II also enlists some other variables for performance comparison.

\section{Solution Method and Control Tuning}

The simulation study of the battery control system is based on analytical solution (35) of problem (P-I) and numerical
TABLE II

Definition of PERFORMANCE VARIABLES

solution of problem (P-IV). To solve problem (P-IV), CVX has been used, which is a MATLAB-based package for specifying and solving convex programs using disciplined convex programming ruleset, see [29] and [30]. The system has been discretized using Euler approximation with sampling interval $h=1 \mathrm{sec}$ and the coolant inlet temperature $T_{f 0}=$ $25{ }^{\circ} \mathrm{C}$. The controller (1-step MPC) has been tuned using first Bryson's rule [31, pg. 537] and then iterative trial and error method to achieve satisfactory balancing performance $\left(\left\|e_{\xi}\right\|_{\infty} \leq 2.5 \%,\left\|e_{T_{s}}\right\|_{\infty} \leq 1{ }^{\circ} \mathrm{C}\right)$ within reasonable time for various drive cycles.

\section{Simulation Results And Discussion}

\section{A. Performance Comparison: US06 Driving}

The balancing performance of UPC and BPC modes of the modular battery has been thoroughly evaluated and compared in simulations. The simulation results for two driving trips of US06 are shown in Fig. 7. The plots are arranged in a $5 \times 3$ matrix of subfigures where columns 2 and 3 correspond to UPC and BPC respectively and each row corresponds to one of five battery performance variables: $v_{L a}(k), \xi(k), T_{s}(k)$, $\left\{\left\|e_{\xi}(k)\right\|_{\infty},\left\|e_{T_{s}}(k)\right\|_{\infty}\right\}$, and $E_{B l \text {,tot }}$. The performance under UDO (uniform duty operation of a conventional battery, see Fig. 1) is shown in column 1 for reference purpose. These plots clearly show that both UPC and BPC significantly reduce SOC deviation among cells relative to the initial condition. Initially, the SOC deviation monotonically decreases almost all the time under both control modes as shown in figures $7(\mathrm{k})$ and 7(1). After decay of initial SOC imbalance, both control modes are able to keep tight equalization of SOCs during both charging and discharging. The temperature deviation under two control modes is significantly lower than that under UDO 
during whole driving despite significant deviation among cell resistances and intensive loading. After decay of initial SOC imbalance, the temperature imbalance remains within $1{ }^{\circ} \mathrm{C}$. This balancing performance is accomplished while simultaneously achieving exact voltage regulation $\left(v_{L a}=v_{L d}\right)$ as shown in the first row of the figure.

The performance statistics are summarized in Table III. The peak cell temperature $T_{s, \text { peak }}$ and mean of highest cell temperature $m_{T_{s, \text { high }}}$ under BPC are considerably less than that under UDO. Therefore, BPC-based modular battery may have longer lifetime than the conventional battery in which unequal cells are equally loaded. The BPC also outperforms UPC in terms of the balancing speed by significant margin. However, it is only marginally better than UPC in terms of mean and standard deviation of balancing errors. In addition, the improvement in the balancing speed and performance variance comes at the cost of some extra energy losses, slightly reduced efficiency $(0.22 \%$ less $)$, and small increase in battery temperature compared to UPC. Since capacity fading is exponential in cell temperature [2], even a small temperature increase over long term under BPC may affect the battery lifetime. Moreover, the BPC-based modular battery requires 2 extra switches inside each module. Therefore, the UPC-based modular battery is a more cost and energy efficient solution without any significant compromise on balancing performance for US06 type driving.

TABLE III

PERFORMANCE COMPARISON UNDER US06 DRIVING

\begin{tabular}{|c|c|c|c|}
\hline Variables & UDO & UPC & BPC \\
\hline$m_{\left\|e_{\xi}\right\|_{\infty}}$ & $6.2 \%$ & $0.37 \%$ & $0.24 \%$ \\
\hline$\sigma_{\left\|e_{\xi}\right\| \infty}$ & $1.0 \%$ & $0.16 \%$ & $0.05 \%$ \\
\hline$m_{\left\|e_{T_{S}}\right\| \infty}$ & $1.00{ }^{\circ} \mathrm{C}$ & $0.52{ }^{\circ} \mathrm{C}$ & $0.36{ }^{\circ} \mathrm{C}$ \\
\hline$\sigma_{\left\|e_{T_{S}}\right\|_{\infty}}$ & $0.33{ }^{\circ} \mathrm{C}$ & $0.06{ }^{\circ} \mathrm{C}$ & $0.04{ }^{\circ} \mathrm{C}$ \\
\hline$m_{T_{s, \text { high }}}$ & $30.76^{\circ} \mathrm{C}$ & $30.17^{\circ} \mathrm{C}$ & $30.35^{\circ} \mathrm{C}$ \\
\hline$\sigma_{T_{s}, \mathrm{high}}$ & $2.02{ }^{\circ} \mathrm{C}$ & $1.70^{\circ} \mathrm{C}$ & $1.76^{\circ} \mathrm{C}$ \\
\hline$T_{s, \text { peak }}$ & $34.9^{\circ} \mathrm{C}$ & $33.6^{\circ} \mathrm{C}$ & $33.8^{\circ} \mathrm{C}$ \\
\hline$m_{T_{B}}$ & $29.82^{\circ} \mathrm{C}$ & $29.77^{\circ} \mathrm{C}$ & $30.06^{\circ} \mathrm{C}$ \\
\hline$E_{B l, \text { tot }}$ & $4.59 W h$ & $4.53 W h$ & $4.81 W h$ \\
\hline $\bar{\eta}_{B}$ & $95.70 \%$ & $95.74 \%$ & $95.48 \%$ \\
\hline$C_{B}$ & $1.91 A h$ & $2.16 A h$ & $2.16 A h$ \\
\hline$T_{b}^{e}$ & - & $152 \mathrm{~s}$ & $108 s$ \\
\hline$T_{b}^{t}$ & - & $418 \mathrm{~s}$ & $220 \mathrm{~s}$ \\
\hline
\end{tabular}

TABLE IV

PERFORMANCE COMPARISON UNDER MOTORWAY DRIVING

\begin{tabular}{l|c|c|c}
\hline Variables & UDO & UPC & BPC \\
\hline$m_{\left\|e_{\xi}\right\|_{\infty}}$ & $6.61 \%$ & $0.52 \%$ & $0.27 \%$ \\
$\sigma_{\left\|e_{\xi}\right\| \infty}$ & $0.74 \%$ & $0.28 \%$ & $0.06 \%$ \\
$m_{\left\|e_{T_{s}}\right\| \infty}$ & $1.11{ }^{\circ} \mathrm{C}$ & $0.69{ }^{\circ} \mathrm{C}$ & $0.40{ }^{\circ} \mathrm{C}$ \\
$\sigma_{\left\|e_{T_{s}}\right\|_{\infty}}$ & $0.38{ }^{\circ} \mathrm{C}$ & $0.15{ }^{\circ} \mathrm{C}$ & $0.07{ }^{\circ} \mathrm{C}$ \\
$m_{T_{s}, \text { high }}$ & $31.00{ }^{\circ} \mathrm{C}$ & $30.64{ }^{\circ} \mathrm{C}$ & $30.88^{\circ} \mathrm{C}$ \\
$\sigma_{T_{s}, \text { high }}$ & $2.02{ }^{\circ} \mathrm{C}$ & $1.92{ }^{\circ} \mathrm{C}$ & $2.00{ }^{\circ} \mathrm{C}$ \\
$T_{s, \text { peak }}$ & $34.28{ }^{\circ} \mathrm{C}$ & $33.38{ }^{\circ} \mathrm{C}$ & $33.61{ }^{\circ} \mathrm{C}$ \\
$m_{T_{B}}$ & $30.00{ }^{\circ} \mathrm{C}$ & $29.97{ }^{\circ} \mathrm{C}$ & $30.46{ }^{\circ} \mathrm{C}$ \\
$E_{B l, \text { tot }}$ & $3.10 \mathrm{Wh}$ & $3.07 \mathrm{Wh}$ & $3.36 \mathrm{Wh}$ \\
$\bar{\eta}_{B}$ & $95.10 \%$ & $95.13 \%$ & $94.75 \%$ \\
$C_{B}$ & $1.91 \mathrm{Ah}$ & $2.16 \mathrm{Ah}$ & $2.16 \mathrm{Ah}$ \\
$T_{b}^{e}$ & - & $76 \mathrm{~s}$ & $72 \mathrm{~s}$ \\
$T_{b}^{t}$ & - & $356 \mathrm{~s}$ & $270 \mathrm{~s}$ \\
\hline \multicolumn{4}{r}{}
\end{tabular}

\section{B. Control Behavior}

The total control actuations under UPC and BPC are shown in Fig. 8 . The plots are arranged in a $2 \times 2$ matrix of subfigures where the first and second columns correspond to control variables under UPC and BPC respectively. The positive and negative control actions $\left[u_{i}^{+}=u_{v i}^{+}+u_{b i}^{+}\right.$and $u_{i}^{-}=u_{b i}^{-}$, see equations (29) and (35)] are displayed in first and second rows respectively. Fig. 8(d) shows that negative control is only slightly engaged by BPC mode to compensate for capacity imbalance. In particular, cells 1 and 2 get some level of negative actuation due to their lower initial dischargeable capacities. The cells 3 and 4 are not negatively actuated as it is not optimal due to their higher resistances. Note that the negative actuation of cells 1 and 2 during driving also reduces after decay of initial SOC imbalance. To better understand the controller working under UPC and BPC modes, all control signal components including the positive voltage control $\left(u_{v i}^{+}\right)$, positive balancing control $\left(u_{b i}^{+}\right)$, and negative balancing control $\left(u_{b i}^{-}\right)$are shown during driving from 50 to 150 seconds in Fig. 9 for cells 1 and 4. The figure shows that Cell $_{1}$ takes extra share of load during high current pulses of short duration to save $\mathrm{Cell}_{4}$ from extra heating whereas Cell 4 takes extra share of low to medium load for long duration to save $\mathrm{Cell}_{1}$ from fast discharging. The feasibility of such kind of load sharing pattern plays a key role to balance SOC and temperature simultaneously.

\section{Performance Comparison: Const. Motorway Driving}

The simulation results for two driving trips on motorway at constant speed of $80 \mathrm{mph}$ are shown in Fig. 10. It is clear from Fig. 10(b) that for constant high load current, the UPC mode struggles to achieve simultaneous thermal and SOC balancing during first trip. It is mainly due to one-to-one coupling between average and rms cell currents under constant loads for UPC mode [see Remark 1 and Fig. 3(c)]. However, during charging after first driving trip, the UPC is able to improve balancing performance. The reversal of current direction plays a main role in this because cells with higher dischargeable (lower chargeable) capacity and higher resistance can now be used less during charging. Moreover, the decrease in current magnitude during charging is also favorable for SOC balancing due to reduced thermal intensity. Nevertheless, the cells (fairly balanced in SOC by the end of charging phase) start deviating again slightly during next driving trip. On the other hand, the BPC shows good thermal and SOC balancing performance independent of current reversal as shown in third column. It is mainly due to relatively loose coupling between average and rms cell currents for BPC mode under constant loads [see Remark 1 and Fig. 3(f)].

The performance statistics are given in Table IV. The BPC balancing performance is quite consistent in terms of mean and standard deviation of balancing errors, but the UPC performance has degraded in this regard relative to that under US06 (compare first four entries of tables III and IV). However, the better balancing performance under BPC comes at the cost of two extra switches per module and some extra energy losses (efficiency reduced by $0.42 \%$ ), which over long term may 

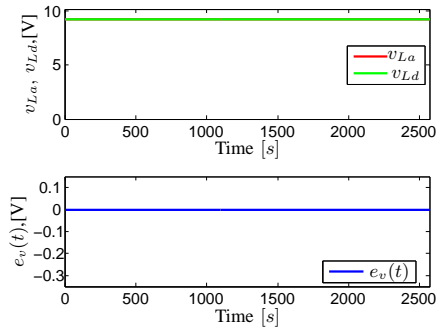

(a)

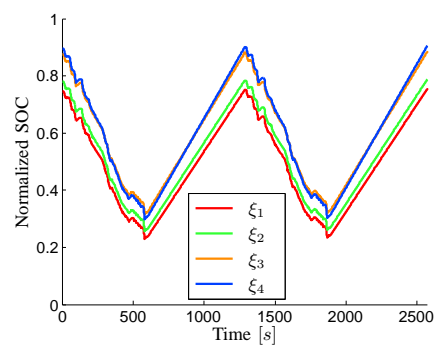

(d)

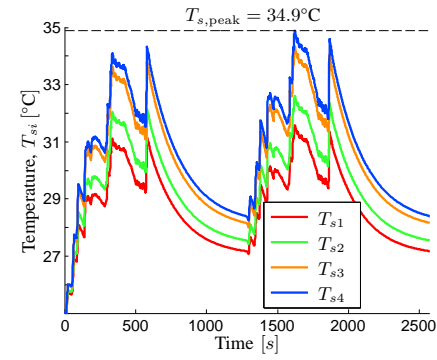

(g)
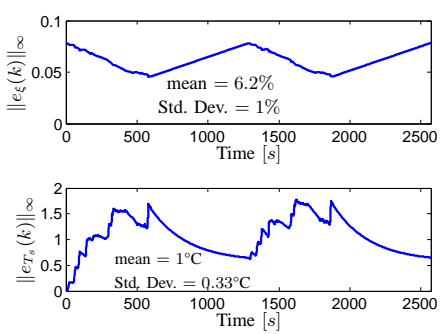

(j)
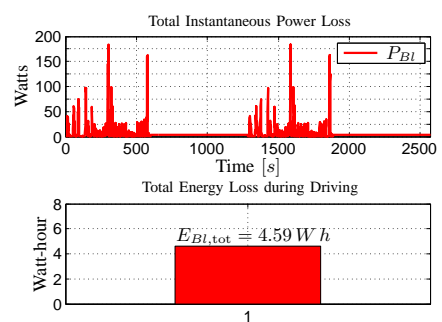

(m)
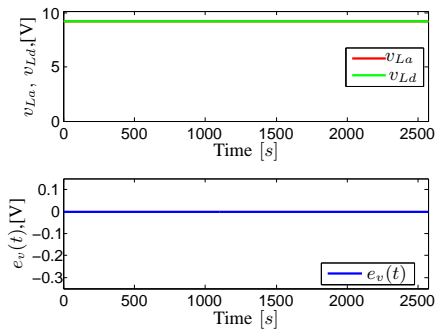

(b)

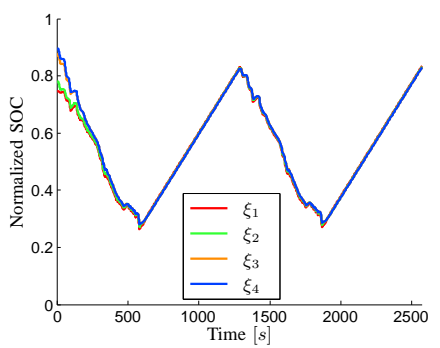

(e)

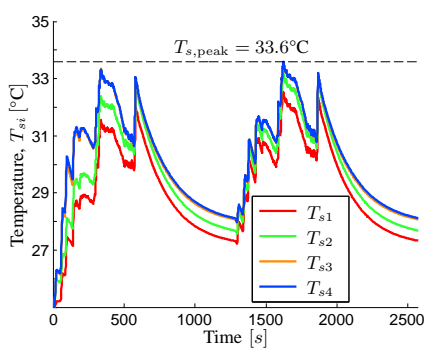

(h)
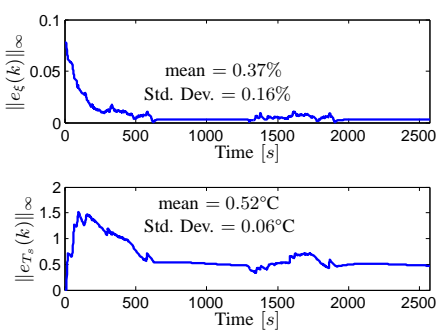

(k)
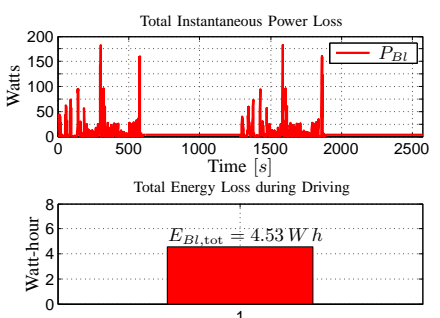

(n)
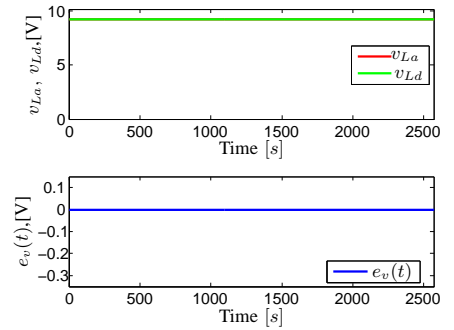

(c)

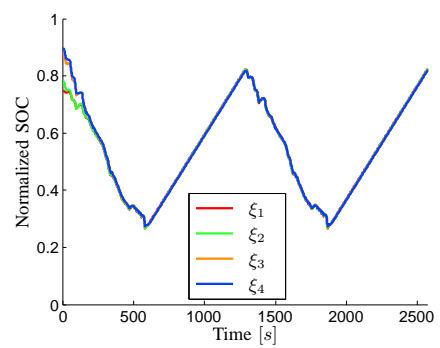

(f)

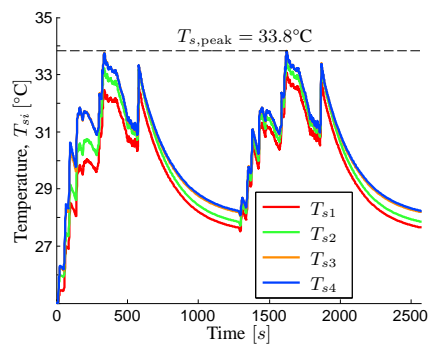

(i)
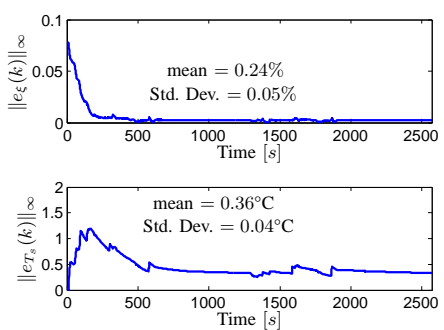

(1)

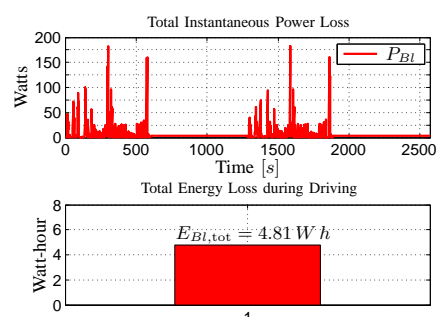

(o)

Fig. 7. Simulation results for control performance under USO6 drive cycle are shown: Uniform Duty Operation (UDO): first column; Unipolar Control Mode $(U P C)$ : second column; and Bipolar Control Mode (BPC): third column. Voltage response and error under (a) UDO, (b) UPC, and (c) BPC. SOC balancing performance under (d) UDO, (e) UPC, and (f) BPC. Thermal balancing performance under (g) UDO, (h) UPC, and (i) BPC. Evolution of balancing errors under (j) UDO, (k) UPC, and (l) BPC. Battery power and energy losses under (m) UDO, (n) UPC, and (o) BPC. 

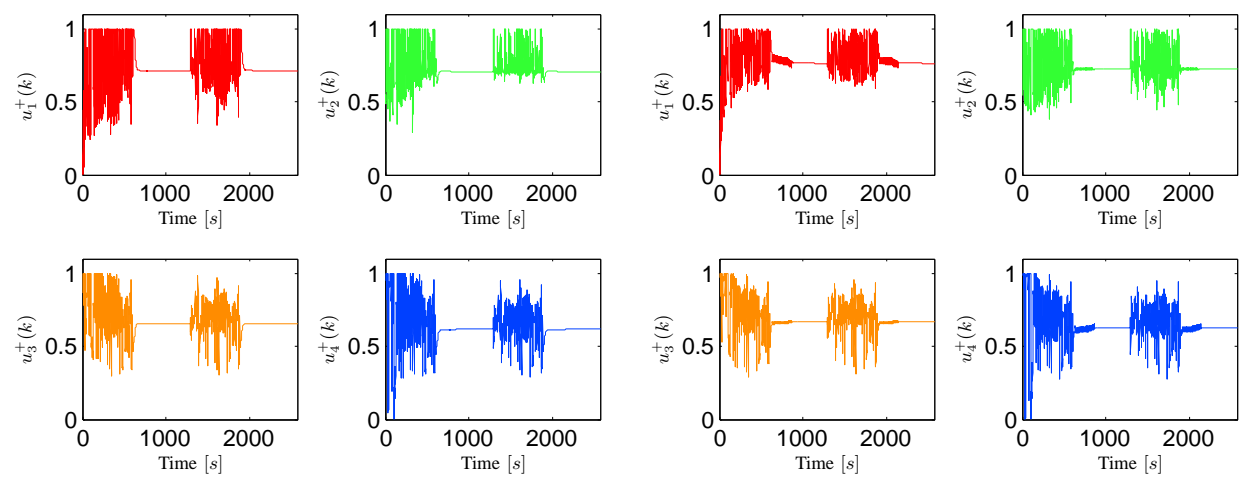

(a)

(b)
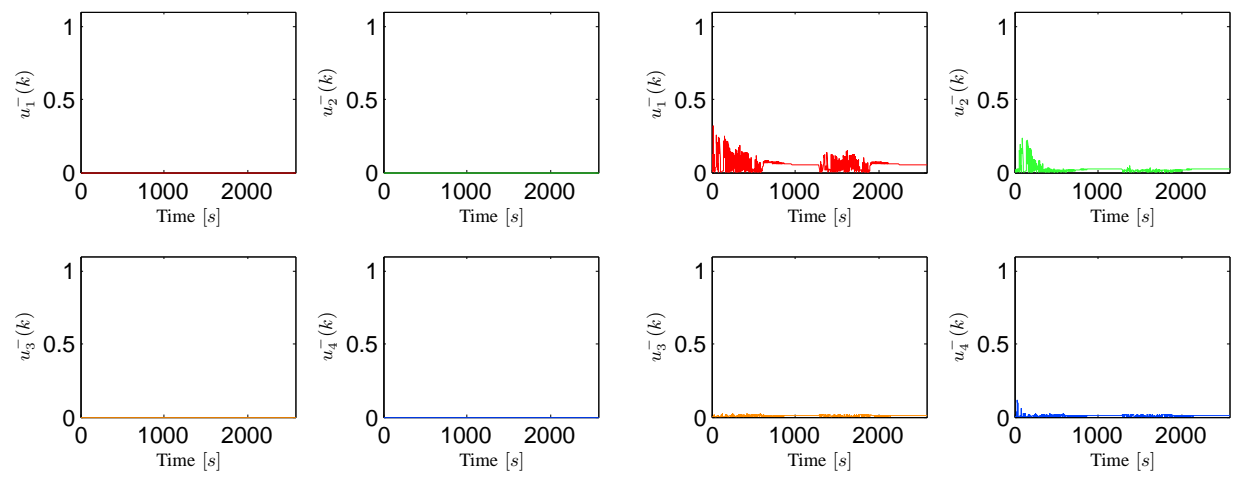

(c)

(d)

Fig. 8. Positive control signals of all cells under (a) UPC and (b) BPC modes. Negative control signals of all cells under (c) UPC and (d) BPC modes.

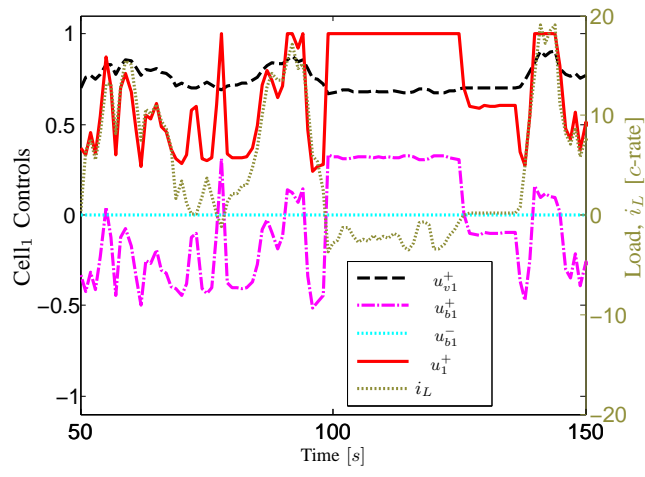

(a)

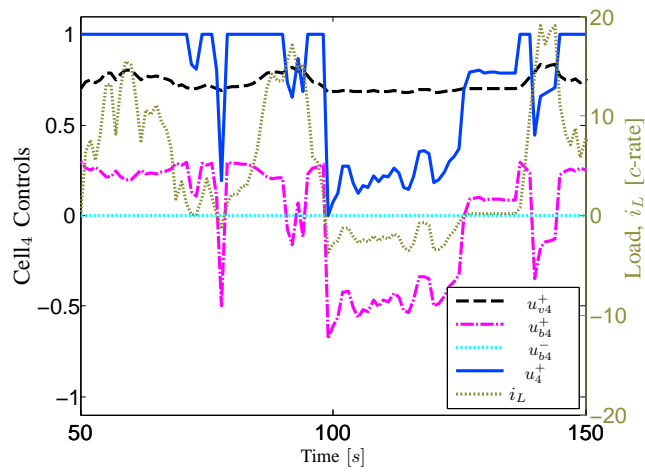

(c)

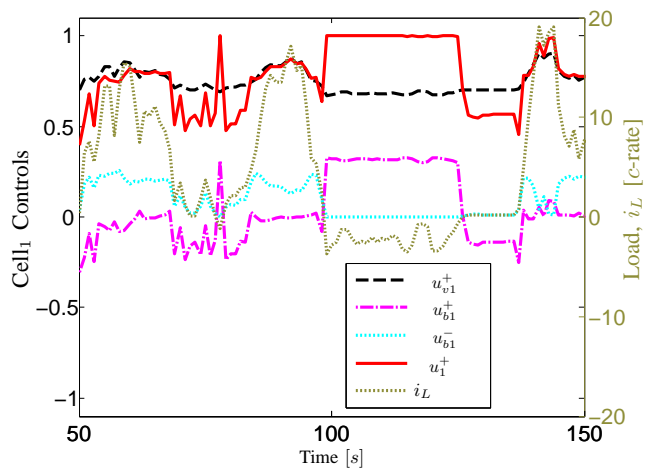

(b)

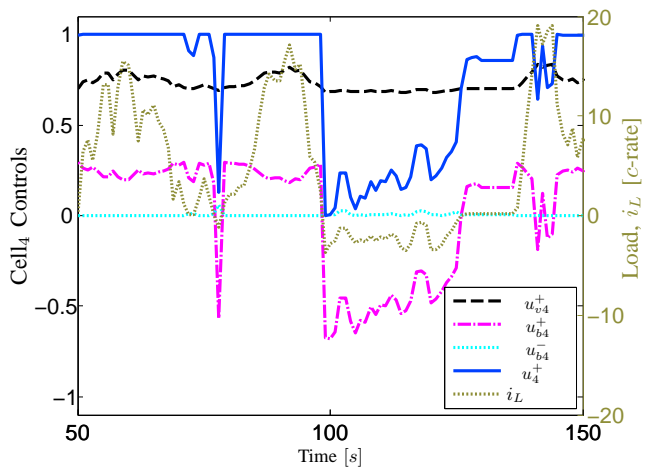

(d)

Fig. 9. Detailed behavior of voltage control, balancing control, and total control signals of cells 1 and 4 along with load current demand $i_{L}$ during driving interval from 50 to 150 second. Cell 1 control signals under (a) UPC and (b) BPC. Cell 4 control signals under (c) UPC and (d) BPC modes. 


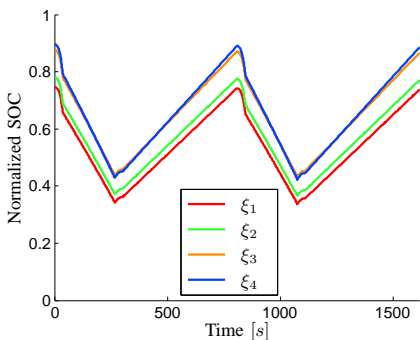

(a)

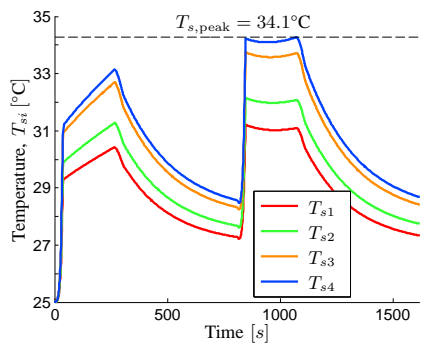

(d)
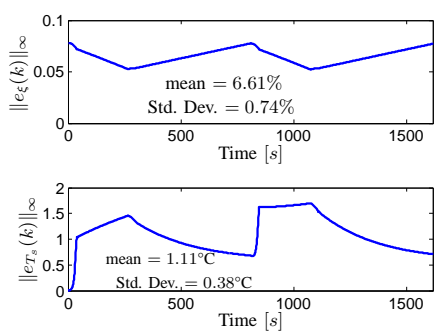

(g)

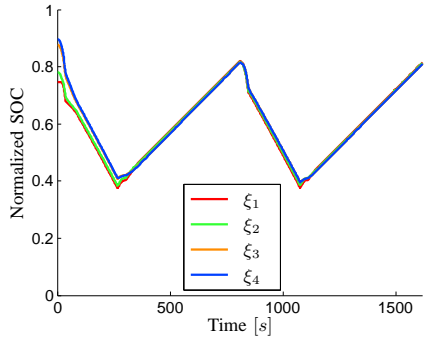

(b)

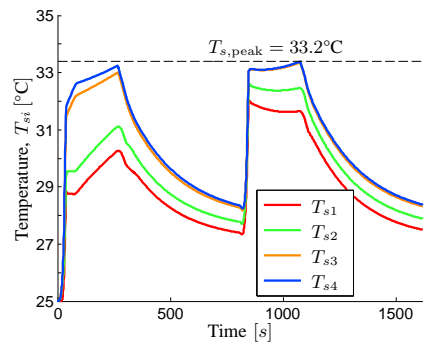

(e)
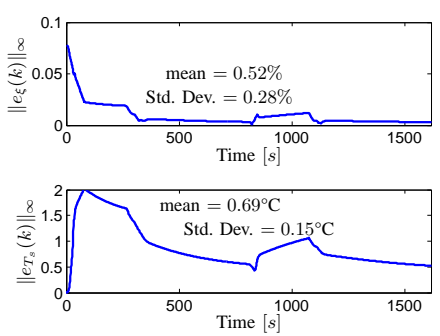

(h)

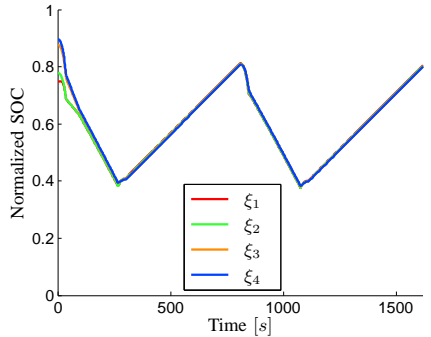

(c)

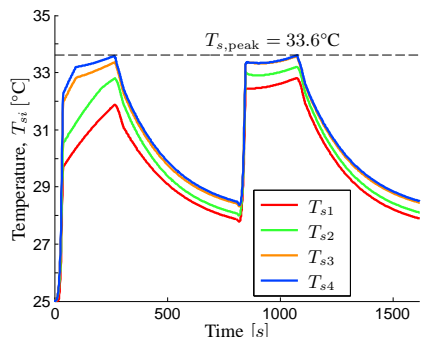

(f)
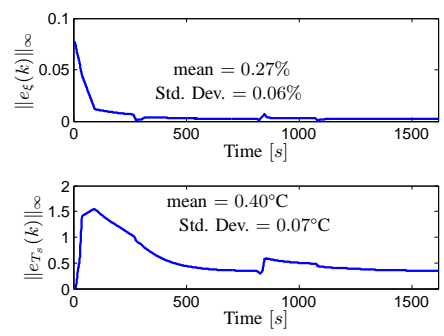

(i)

Fig. 10. Simulation results for control performance under Constant Speed drive cycle are shown: Uniform Duty Operation (UDO): first column; Unipolar Control Mode (UPC): second column; and Bipolar Control Mode (BPC): third column. SOC balancing performance under (a) UDO, (b) UPC, and (c) BPC. Thermal balancing performance under (d) UDO, (e) UPC, and (f) BPC. Evolution of balancing errors under (g) UDO, (h) UPC, and (i) BPC.

reduce battery life-time. Moreover, the BPC gives significant benefit in SOC balancing particularly during first driving trip, but this benefit is only marginal after start of external charging phase. In addition, the UPC performs significantly better than UDO in terms of all statistics. Therefore, the UPC-based modular battery is still an acceptable solution.

\section{SUMmARY AND CONCLUSIONS}

In this paper, the bipolar control (BPC) of a modular battery for simultaneous thermal and SOC balancing has been presented. Its balancing performance has been thoroughly compared with unipolar control (UPC) mode that was introduced in our earlier study [16]. The BPC mode allows polarity inversion (so-called negative actuation) of cells in the string, but needs full-bridge converter. The UPC mode does not allow polarity inversion, but only needs a half-bridge converter. The averaged model of a switched modular battery has been derived in a general setting, which resulted in the formulation of convex control problems under both modes. The predictive control method employed in these two modes is tailored based on a controller structure proposed in [16]. In this method, the controller is decomposed into two orthogonal components, one for voltage control and the other for balancing control.

The performance comparison between UPC and BPC has been shown particularly for US06 and constant $80 \mathrm{mph}$ mo- torway driving. These driving cycles are more challenging for simultaneous balancing of temperature and SOC due to their aggressive nature (high $c$-rate) and lower level of load variations compared to stop-n-go urban driving. The results show that BPC gives more consistent balancing performance that is independent of variation in magnitude and direction of load current. This becomes possible due to the feasibility of negative cell actuations, which results in loose coupling between average and rms values of cell current, giving some extra freedom to control temperature and SOC. It is also noteworthy that the need of negative actuations reduces after initial balancing phase. The balancing performance of UPC during first trip of US06 driving is not as good as BPC. However, looking over full charge/discharge cycle, there is only a marginal difference in performance. This is due to reversal of current direction during charging phase, which facilitates the cell balancing task for UPC. The performance of UPC degrades to some extent particularly during constant high speed motor way driving. The performance recovers during subsequent charging phase, but then slightly degrades again during next trip. Therefore, the UPC struggles without variation in current magnitude and results in somewhat higher variance in performance compared to that under BPC.

However, the better balancing performance of BPC comes at the cost of slightly reduced battery efficiency due to extra 
losses during negative actuation of cells, which increases battery temperature. Although the temperature rise is small, it is better to avoid it because cell ageing is exponential in temperature. The BPC mode also needs $2 n(n=$ no. of modules) extra switches, which implies higher cost and semiconductor losses. In addition, the balancing performance of UPC does not degrade drastically if external charging can be provided after each short driving trip, which is possible at least for EV and PHEV applications. Therefore, looking over multiple charge/discharge cycles in such applications, the UPC mode is a more cost-effective solution without any significant compromise on balancing performance. The BPC, on the other hand, may show some merit particularly in applications, which require high load current pulses of long duration and have no dedicated external charging as in HEVs.

\section{APPENDIX}

The matrices for model (21a)-(21b) are given by

$$
\begin{aligned}
& A=\left[\begin{array}{cc}
A_{E} & 0 \\
0 & A_{\vartheta}
\end{array}\right], B\left(i_{L}(t)\right)=\left[\begin{array}{cc}
B_{E} i_{L} & 0 \\
0 & B_{\vartheta} i_{L}^{2}
\end{array}\right] M_{3}, \\
& A_{E}=0_{n \times n}, B_{E}=-\operatorname{diag}\left(b_{e 1}, \cdots, b_{e n}\right) \in \mathbb{R}^{n \times n} \text {, } \\
& A_{\vartheta}=\left[\begin{array}{cc}
A_{T} & W_{T} \\
0_{n}^{\mathrm{T}} & 0
\end{array}\right], \quad B_{\vartheta}=\left[\begin{array}{c}
B_{T} \\
0_{n}^{\mathrm{T}}
\end{array}\right], \quad M_{3}=\left[\begin{array}{cc}
I_{n} & -I_{n} \\
I_{n} & I_{n}
\end{array}\right], \\
& A_{T}=\left[a_{t i j}\right] \in \mathbb{R}^{n \times n}, \quad B_{T}=\operatorname{diag}\left(b_{t 1}, \cdots, b_{t n}\right) \in \mathbb{R}^{n \times n}, \\
& W_{T}=\left[\begin{array}{lll}
w_{t 1} & \cdots & w_{t n}
\end{array}\right]^{\mathrm{T}} \in \mathbb{R}^{n}, \\
& C=\left[\begin{array}{cc}
0 & I_{n+1} \\
0_{n}^{\mathrm{T}} & 0_{n+1}^{\mathrm{T}}
\end{array}\right], D\left(i_{L}(t)\right)=\left[\begin{array}{c}
0 \\
D_{v}(t)
\end{array}\right], \\
& D_{v}(t)=\left[\begin{array}{ll}
D_{v}^{+}(t) & -D_{v}^{-}(t)
\end{array}\right] \in \mathbb{R}^{1 \times 2 n} \text {, }
\end{aligned}
$$

where $A_{T}$ is a constant lower triangular thermal subsystem matrix and the coefficients $a_{t i j}$ and $w_{t i}$ are thermal circuit parameters for coolant flow from Cell ${ }_{1}$ towards Cell ${ }_{i}$. The coefficients $b_{e i}=\frac{1}{3600 C_{e i}}$ and $b_{t i}=\frac{R_{e i}}{C_{s i}}$. Note that $D_{v}$ is a direct feedthrough gain from control $u$ to terminal voltage $v_{L a}$ and $D_{v}^{+}$and $D_{v}^{-}$are defined in (18a) and (18b) respectively.

\section{REFERENCES}

[1] J. Vetter, P. Novak, M. Wagner, and et.el., "Ageing mechanisms in lithium-ion batteries," Journal of power sources, vol. 147, no. 1, pp. 269-281, 2005.

[2] J. Wang, P. Liu, Hicks-Garner, and et.el., "Cycle-life model for graphite$\mathrm{LiFePO}_{4}$ cells," Journal of Power Sources, vol. 196, no. 8, pp. 3942 3948, 2011.

[3] T. M. Bandhauer, S. Garimella, and T. F. Fuller, "A critical review of thermal issues in lithium-ion batteries," Journal of the Electrochemical Society, vol. 158, no. 3, pp. R1-R25, 2011.

[4] J. Groot, State-of-Health Estimation of Li-ion Batteries: Ageing Models, ser. PhD Thesis. New Series, no: 3815. Chalmers University of Technology, 2014.

[5] L. Lu, X. Han, J. Li, J. Hua, and M. Ouyang, "A review on the key issues for lithium-ion battery management in electric vehicles," Journal of Power Sources, vol. 226, pp. 272-288, 2013.

[6] F. Altaf, L. Johannesson, and B. Egardt, "Simultaneous Thermal and State-of-Charge Balancing of Batteries: A Review," in Vehicle Power and Propulsion Conference (VPPC), 2014 IEEE, Oct. 2014, pp. 1-7.

[7] M. Dubarry, N. Vuillaume, and B. Y. Liaw, "Origins and accommodation of cell variations in li-ion battery pack modeling," International Journal of Energy Research, vol. 34, no. 2, pp. 216-231, 2010.

[8] R. Mahamud and C. Park, "Reciprocating air flow for li-ion battery thermal management to improve temperature uniformity," Journal of Power Sources, vol. 196, no. 13, pp. 5685 - 5696, 2011.

[9] J. Gallardo-Lozano, Romero-Cadaval, and et.el., "Battery equalization active methods," Journal of Power Sources, vol. 246, pp. 934-949, 2014
[10] J. Cao, N. Schofield, and A. Emadi, "Battery balancing methods: A comprehensive review," in Vehicle Power and Propulsion Conference, 2008. VPPC '08. IEEE, sept. 2008, pp. $1-6$.

[11] M. Einhorn, W. Guertlschmid, T. Blochberger, R. Kumpusch, R. Permann, F. Conte, C. Kral, and J. Fleig, "A current equalization method for serially connected battery cells using a single power converter for each cell," Vehicular Technology, IEEE Transactions on, vol. 60, no. 9, pp. 4227-4237, Nov 2011.

[12] F. Altaf, L. Johannesson, and B. Egardt, "On Thermal and State-ofCharge Balancing using Cascaded Multi-level Converters," Journal of Power Electronics, vol. 13, no. 4, pp. 569-583, July 2013.

[13] _ "Evaluating the Potential for Cell Balancing using a Cascaded Multi-Level Converter using Convex Optimization," in IFAC Workshop on Engine and Powertrain Control, Simulation and Modeling, 2012, Oct. 2012.

[14] F. Altaf, Thermal and State-of-Charge Balancing of Batteries using Multilevel Converters. Licentiate Thesis, Chalmers University of Technology, 2014. [Online]. Available: http://publications.lib.chalmers. se/records/fulltext/194660/194660.pdf

[15] J. Barreras, C. Pinto, and et.al., "Multi-objective control of balancing systems for li-ion battery packs: A paradigm shift?" in Vehicle Power and Propulsion Conference (VPPC), 2014 IEEE, Oct. 2014.

[16] F. Altaf, B. Egardt, and L. Johannesson, "Load Management of Modular Battery using Model Predictive Control: Thermal and State-of-Charge Balancing," Control Systems Technology, IEEE Transactions on, to appear in 2016, preprint available at http://publications.lib.chalmers.se/ records/fulltext/228087/local_228087.pdf.

[17] — "Electro-thermal Control of Modular Battery using Model Predictive Control with Control Projections," in IFAC Workshop on Engine and Powertrain Control, Simulation and Modeling, 2015, vol. 48, no. 15, Aug. 2015, pp. 368 - 375 .

[18] A. Manenti, A. Abba, A. Merati, S. Savaresi, and A. Geraci, "A new bms architecture based on cell redundancy," Industrial Electronics, IEEE Transactions on, vol. 58, no. 9, pp. 4314-4322, 2011.

[19] W. Huang and J. Abu Qahouq, "Energy sharing control scheme for state-of-charge balancing of distributed battery energy storage system," Industrial Electronics, IEEE Transactions on, vol. 62, no. 5, pp. 2764 2776, May 2015.

[20] M. A. Xavier and M. S. Trimboli, "Lithium-ion battery cell-level control using constrained model predictive control and equivalent circuit models," Journal of Power Sources, vol. 285, pp. 374 - 384, 2015.

[21] Z. Zheng, K. Wang, L. Xu, and Y. Li, "A hybrid cascaded multilevel converter for battery energy management applied in electric vehicles,' Power Electronics, IEEE Transactions on, vol. 29, no. 7, pp. 3537-3546, July 2014.

[22] T. Soong and P. Lehn, "Evaluation of emerging modular multilevel converters for bess applications," Power Delivery, IEEE Transactions on, vol. 29, no. 5, pp. 2086-2094, Oct 2014.

[23] X. Hu, S. Li, and H. Peng, "A comparative study of equivalent circuit models for li-ion batteries," Journal of Power Sources, vol. 198, no. 0, pp. $359-367,2012$

[24] X. Lin, H. E. Perez, S. Mohan, J. B. Siegel, A. G. Stefanopoulou, Y. Ding, and M. P. Castanier, "A lumped-parameter electro-thermal model for cylindrical batteries," Journal of Power Sources, vol. 257, pp. $1-11,2014$.

[25] X. Lin, H. Fu, H. E. Perez, and et.el., "Parameterization and observability analysis of scalable battery clusters for onboard thermal management," Oil \& Gas Science and Technology-Revue d'IFP Energies nouvelles, vol. 68 , no. 1 , pp. $165-178,2013$.

[26] X. Lin, H. Perez, J. Siegel, A. Stefanopoulou, and et.el., "Online parameterization of lumped thermal dynamics in cylindrical lithium ion batteries for core temperature estimation and health monitoring," Control Systems Technology, IEEE Transactions on, vol. 21, no. 5, pp. 17451755 , Sept 2013.

[27] K. Wipke, M. Cuddy, and S. Burch, "Advisor 2.1: a user-friendly advanced powertrain simulation using a combined backward/forward approach," Vehicular Technology, IEEE Transactions on, vol. 48, no. 6, pp. 1751-1761, Nov 1999.

[28] L. Zhong, C. Zhang, Y. He, and Z. Chen, "A method for the estimation of the battery pack state of charge based on in-pack cells uniformity analysis," Applied Energy, vol. 113, pp. 558-564, 2014.

[29] S. Boyd and L. Vandenberghe, Convex Optimization. Cambridge University Press, 2006.

[30] M. Grant and S. Boyd, "CVX: Matlab software for disciplined convex programming, version 1.21," Apr 2011.

[31] G. F. Franklin, J. D. Powell, and A. Emami-Naeini, Feedback control of dynamics systems, 4th ed. Prentice Hall, NJ, 2002. 


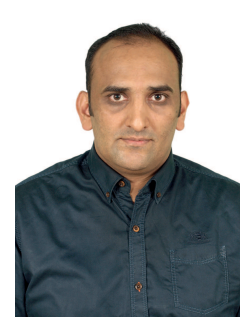

Faisal Altaf received the B.E. degree in mechatronics from the National University of Sciences and Technology (NUST), Islamabad, Pakistan, in 2004, the M.Sc. degree in electrical engineering from the KTH Royal Institute of Technology, Stockholm, Sweden, in 2011, and the Ph.D. degree in automatic control from Chalmers University of Technology, Gothenburg, Sweden, in 2016.

$\mathrm{He}$ worked as a R\&D/Control Engineer with $\mathrm{Mi}-$ crowave Engineering Research Laboratory at NUST, from 2004 to 2008, where he was heavily involved in the development of a radar system. From 2010 to 2011, he was a Research Engineer in the Department of Automatic Control at KTH where he was involved in control over wireless sensor networks. His current research interests are at the intersection of constrained optimal control, convex optimization, estimation, batteries, and power electronics with special focus on model predictive control and battery management systems in the automotive area.

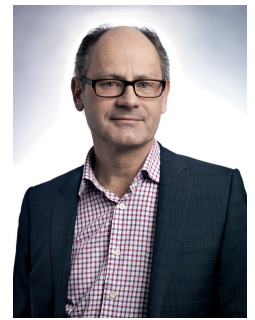

Bo Egardt received the M.Sc. degree in electrical engineering and the Ph.D. degree in automatic control from Lund Institute of Technology, Sweden, in 1974 and 1979, respectively.

He was a Research Associate at the Information Systems Laboratory, Stanford, CA, USA, in 1980. From 1981 to 1989, he was with Asea Brown Boveri, Västerås, Sweden, where he was heavily involved in the introduction of adaptive control in the process industry. In 1989, he was appointed as a Professor of Automatic Control with the Chalmers University of Technology, Gothenburg, Sweden. His current research interests include adaptive and hybrid control and applications of control in the automotive area.

Dr. Egardt is a member of the Royal Swedish Academy of Engineering Sciences, member of the editorial board for the International Journal of Adaptive Control and Signal Processing, and a Fellow of the IEEE. He was an Associate Editor of the IEEE Transactions on Control Systems Technology and of the European Journal of Control. 